\title{
Methylotrophy, alkane-degradation, and pigment production as defining features of the globally distributed yet-uncultured phylum Binatota
}

\author{
Chelsea L. Murphy ${ }^{1}$, Peter F. Dunfield ${ }^{2}$, Andriy Sheremet ${ }^{2}$, John R. Spear ${ }^{3}$, Ramunas \\ Stepanauskas $^{4}$, Tanja Woyke ${ }^{5}$, Mostafa S. Elshahed ${ }^{1}$, and Noha H. Youssef ${ }^{1 *}$ \\ ${ }^{1}$ Department of Microbiology and Molecular Genetics, Oklahoma State University, Stillwater, \\ OK, USA, ${ }^{2}$ Department of Biological Sciences, University of Calgary, Calgary, AB, Canada, \\ ${ }^{3}$ Civil and Environmental Engineering, Colorado School of Mines, Golden, Colorado, USA ${ }^{4}$ \\ Bigelow Laboratory for Ocean Sciences, East Boothbay, Maine, USA, and ${ }^{5}$ Department of \\ Energy Joint Genome Institute, Berkley, California, USA
}

*Correspondence: Noha Youssef: Noha@okstate.edu 


\section{Abstract}

2 The recent leveraging of genome-resolved metagenomics has opened a treasure trove of genomes

3 from novel uncultured microbial lineages, yet left many clades undescribed. We here present a

4 global analysis of genomes belonging to the Binatota (UBP10), a globally distributed, yet-

5 uncharacterized bacterial phylum. All orders in the Binatota encoded the capacity for aerobic

6 methylotrophy using methanol, methylamine, sulfomethanes, chloromethanes, and potentially

7 methane as substrates. Methylotrophy in the Binatota was characterized by order-specific

8 substrate degradation preferences, as well as extensive metabolic versatility, i.e. the utilization of

9 diverse sets of genes, pathways and combinations to achieve a specific metabolic goal. The

10 genomes also encoded an arsenal of alkane hydroxylases and monooxygenases, potentially

11 enabling growth on a wide range of alkanes and fatty acids. Pigmentation is inferred from a

12 complete pathway for carotenoids (lycopene, $\beta$ and $\gamma$ carotenes, xanthins, chlorobactenes, and

13 spheroidenes) production. Further, the majority of genes involved in bacteriochlorophyll $a, c$,

14 and $d$ biosynthesis were identified; although absence of key genes and failure to identify a

15 photosynthetic reaction center precludes proposing phototrophic capacities. Analysis of 16S

16 rRNA databases showed Binatota's preferences to terrestrial and freshwater ecosystems,

17 hydrocarbon-rich habitats, and sponges supporting their suggested potential role in mitigating

18 methanol and methane emissions, alkanes degradation, and nutritional symbiosis with sponges.

19 Our results expand the lists of methylotrophic, aerobic alkane degrading, and pigment-producing

20 lineages. We also highlight the consistent encountering of incomplete biosynthetic pathways and

21 gene shrapnel in microbial genomes, a phenomenon necessitating careful assessment when

22 assigning putative functions based on a set-threshold of pathway completion. 


\section{Introduction}

25 Approaches that directly recover genomes from environmental samples and bypass the hurdle of

26 cultivation (single-cell genomics and genome-resolved metagenomics) have come of age in the

27 last decade. The new availability of environmentally sourced genomes, obtained as SAGs (single

28 amplified genomes) or MAGs (metagenome-assembled genomes) is having a lasting impact on

29 the field of microbial ecology. Distinct, yet often complementary and intertwined, strategies are

30 employed for the analysis of the deluge of obtained genomes. Site- or habitat-specific studies

31 focus on spatiotemporal sampling of a single site or habitat of interest. The obtained genomes are

32 then analyzed to elucidate how resident taxa mediate substrates turnover and elemental cycling,

33 examine microbial interactions on the metabolic and cellular levels, and document how the

34 community responds to natural and anthropogenic changes ${ }^{1,2,3,4}$. Function-based studies focus

35 on genomes from single or multiple habitats to identify and characterize organisms involved in a

36 specific process, e.g. cellulose degradation ${ }^{5}$ or sulfate-reduction ${ }^{6}$. Phylogeny-oriented

37 (phylocentric) studies, on the other hand, focus on characterizing genomes belonging to a

38 specific lineage of interest, with the aim of delineating its pan, core, and dispensable gene

39 repertoire, documenting the defining metabolic capabilities and physiological preferences for the

40 entire lineage and encompassed clades ${ }^{7,8}$, understanding the lineage's ecological distribution

41 and putative roles in various habitats ${ }^{4,9}$, and elucidating genomic basis underpinning niche

42 specializing patterns ${ }^{10}$. The scope of phylocentric studies could range from the analysis of a

43 single genome from a single ecosystem ${ }^{11}$, to global sampling and in-silico analysis efforts ${ }^{12,13}$.

44 The feasibility and value of phylocentric strategies have recently been enhanced by the

45 development of a genome-based (phylogenomic) taxonomic outline based on extractable data

46 from MAGs and SAGs providing a solid framework for knowledge building and data 
47 communication ${ }^{14}$, as well as recent efforts for massive, high-throughput binning of genomes

48 from global collections of publicly available metagenomes in GenBank nr and Integrated

49 Microbial Genomes \& Microbiomes (IMG/M) databases ${ }^{15,16}$.

50 Candidate phylum UBP10 has originally been described as one of the novel lineages

51 recovered from a massive binning effort that reconstructed thousands of genomes from publicly

52 available metagenomic datasets ${ }^{15}$. UBP10 has subsequently been named candidate phylum

53 Binatota (henceforth Binatota) in an effort to promote nomenclature for uncultured lineages

54 based on attributes identified in MAGs and SAGs ${ }^{17}$. The recent generation of 52,515 distinct

55 MAGs binned from over 10,000 metagenomes ${ }^{16}$ has greatly increased the number of available

56 Binatota genomes. Here, we utilize a phylocentric approach and present a comparative analysis

57 of the putative metabolic and biosynthetic capacities and putative ecological roles of members of

58 the candidate phylum Binatota, as based on sequence data from 108 MAGs. Our study

59 documents aerobic methylotrophy, aerobic alkane degradation, and carotenoid pigmentation as

60 defining traits in the Binatota. We also highlight the presence of incomplete chlorophyll

61 biosynthetic pathways in all genomes, and propose several evolutionary-grounded scenarios that

62 could explain such pattern. 


\section{Results}

65 Overview. A total of 108 Binatota MAGs with $>70 \%$ completion and $<10 \%$ contamination were

66 used for this study, which included 86 medium-quality and 22 high-quality genomes, as based on

67 MIMAG standards ${ }^{18}$. Binatota genomes clustered into seven orders designated as Bin18 $(\mathrm{n}=2)$,

68 Binatales $(n=48), \operatorname{HRBin} 30(n=7), \operatorname{UBA} 1149(n=9), \operatorname{UBA} 9968(n=34), \operatorname{UBA} 12015(n=1)$,

69 UTPRO1 ( $\mathrm{n}=7$ ), encompassing12 families, and 24 genera (Figure 1, Table S1). 16S rRNA gene

70 sequences extracted from orders Bin18 and UBA9968 genomes were classified in SILVA

71 (release 138) ${ }^{19}$ as members of class bacteriap25 in the phylum Myxococcota, order Binatales

72 and order HRBin30 as uncultured phylum RCP2-54, and orders UBA1149 and UTPRO1 as

73 uncultured Desulfobacterota classes (Table S1). RDP II-classification (July 2017 release,

74 accessed July 2020) classified all Binatota sequences as unclassified Deltaproteobacteria (Table

$75 \mathrm{~S} 1)$.

76 Methylotrophy in the Binatota.

77 1. C1 substrates oxidation to formaldehyde.

78 Methanol: With the exception of HRBin30, all orders encoded at least one type of methanol

79 dehydrogenase (Figure 2a). Three distinct types of methanol dehydrogenases were identified

80 (Figure 2a, b): 1. The NAD(P)-binding MDO/MNO-type methanol dehydrogenase (mno),

81 typically associated with Gram-positive methylotrophic bacteria (Actinobacteria and Bacillus

82 methanolicus) ${ }^{20}$, was the only type of methanol dehydrogenase identified in orders UBA9968,

83 UBA12105, and UTPR01 (Figure 2a, Extended data 1), as well as some UBA1149 and Binatales

84 genomes. 2. The MDH2-type methanol dehydrogenase, previously discovered in members of the

85 Burkholderiales and Rhodocyclales ${ }^{21}$, was encountered in the majority of order UBA1149

86 genomes and in two Binatales genomes, and 3. The lanthanide-dependent pyrroloquinoline 
87 quinone (PQQ) methanol dehydrogenase XoxF-type was encountered in nine genomes from the 88 orders Bin18, and Binatales, together with the accessory XoxG (c-type cytochrome) and XoxJ

89 (periplasmic binding) proteins (Figure 2a). All later genomes also encoded PQQ biosynthesis.

90 Surprisingly, none of the genomes encoded the MxaF1-type (MDH1) methanol dehydrogenase,

91 typically encountered in model methylotrophs ${ }^{22}$.

92 Methylamine: All Binatota orders except UBA9968 encoded methylamine degradation capacity.

93 The direct periplasmic route (methylamine dehydrogenase; mau) was more common, with mauA

94 and mauB enzyme subunits encoded in the Binatales, HRBin30, UBA1149, UBA12105, and

95 UTPR01 (Figure 2a, Extended data 1). Amicyanin (encoded by mauC) is the most probable

96 electron acceptor for methylamine dehydrogenase ${ }^{22}$ (Figure 2a). On the other hand, one Bin18

97 genome, and two Binatales genomes (that also encode the mau cluster) encoded the full

98 complement of genes for methylamine oxidation via the indirect glutamate pathway (Figure 2a,

99 Extended data 1).

100 Methylated sulfur compounds: Binatota genomes encoded several enzymes involved in the

101 degradation of dimethyl sulfone, methane sulfonic acid (MSA), and dimethyl sulfide (DMS).

102 Nine genomes (two Bin18, and 7 Binatales) encoded dimethyl sulfone monooxygenase (sfnG)

103 involved in the degradation of dimethyl sulfone to MSA with the concomitant release of

104 formaldehyde. Three of these nine genomes also encoded alkane sulfonic acid monooxygenase

105 (ssuD), which will further degrade the MSA to formaldehyde and sulfite. Degradation of DMS

106 via DMS monooxygenase $(d m o A)$ to formaldehyde and sulfide was encountered in 13 genomes

107 (2 Bin18, 9 Binatales, and 2 UBA9968). Further, one Binatales genome encoded the $d s o$ system

108 (EC: 1.14.13.245) for DMS oxidation to dimethyl sulfone, which could be further degraded to

109 MSA as explained above (Figure 2a, Extended data 1). 
110 Dihalogenated methane: One Bin18 genome encoded the specific dehalogenase/ glutathione S-

111 transferase $(\operatorname{dcm} A)$ capable of converting dichloromethane to formaldehyde.

112 Methane: Genes encoding particulate methane monooxygenase (pMMO) were identified in

113 orders Bin18 (2/2 genomes) and Binatales (9/48 genomes) (Figure 2a, Extended data 1), while

114 genes encoding soluble methane monooxygenase (sMMO) were not found. A single copy of all

115 three pMMO subunits (A, B, and C) was encountered in 9 of the 11 genomes, while two copies

116 were identified in two genomes. pMMO subunit genes (A, B, and $\mathrm{C}$ ) occurred as a contiguous

117 unit in all genomes, with a $\mathrm{CAB}$ (5 genomes), and/or CAxB or $\mathrm{CAxxB}$ (8 genomes, where $\mathrm{x}$ is a

118 hypothetical protein) organization, similar to the pMMO operon structure in methanotrophic

119 Proteobacteria, Verrucomicrobia, and Candidatus Methylomirabilis (NC10) ${ }^{23,24,25,26}$ (Figure

120 2c) ). In addition, five of the above eleven genomes also encoded a $p m o D$ subunit, recently

121 suggested to be involved in facilitating the enzyme complex assembly, and/or in electron transfer

122 to the enzyme's active site ${ }^{27,28}$. Phylogenetic analysis of Binatota $p m o A$ sequences revealed

123 their affiliation with two distinct clades: the yet-uncultured Cluster 2 TUSC (ropical Uppland

124 Soil Cluster) methanotrophs ${ }^{29}$ (2 Binatales genomes), and a clade encompassing pmo $A$

125 sequences from Actinobacteria (Nocardioides sp. strain CF8, Mycolicibacterium, and

126 Rhodococcus) and SAR324 (Candidatus Lambdaproteobacteria) ${ }^{30,31}$ (Figure 2d). Previous

127 studies have linked Cluster 2 TUSC pMMO-harboring organisms to methane oxidation based on

128 selective enrichment on methane in microcosms derived from Lake Washington sediments ${ }^{32}$. All

129 Binatota genomes encoding TUSC-affiliated pMMO, also encoded genes for downstream

130 methanol and formaldehyde oxidation as well as formaldehyde assimilation (see below),

131 providing further evidence for their putative involvement in methane oxidation. On the other

132 hand, studies on Nocardioides sp. strain CF8 demonstrated its capacity to oxidize short chain 
133 (C2-C4) hydrocarbons, but not methane, via its pMMO, and its genome lacked methanol

134 dehydrogenase homologues ${ }^{33}$. Such data favor a putative short chain hydrocarbon degradation

135 function for organisms encoding this type of pMMO, although we note that five out of the nine

136 Binatota genomes encoding SAR324/ Actinobacteria-affiliated $p m o A$ sequences also encoded at

137 least one methanol dehydrogenase homologue. Modeling pMMO subunits from both TUSC-type

138 and Actinobacteria/SAR324-type Binatota genomes using Methylococcus capsulatus (Bath) 3D

139 model (PDB ID: 3rbg) revealed a heterotrimeric structure $\left(\alpha_{3} \beta_{3} \gamma_{3}\right)$ with the 7,2 , and 5 alpha

140 helices of the PmoA, PmoB, and PmoC subunits, respectively, as well as the beta sheets

141 characteristic of PmoA, and PmoB subunits (Figure 2e). Modeling also predicted binding

142 pockets of the dinuclear $\mathrm{Cu}$ ions and $\mathrm{Zn}$ ligands (Figure 2e).

143 2. Formaldehyde oxidation to $\mathrm{CO}_{2}$ : Three different routes for formaldehyde oxidation to

144 formate were identified (Figure 3). First, the Actinobacteria specific thiol-dependent

145 formaldehyde dehydrogenase $(f a d h / m s c R)(E C: 1.1 .1 .306)$ was, surprisingly, detected in the

146 majority (96 out of 108) of genomes (Figure 3a, Extended data 1). The enzyme requires a

147 specific thiol (mycothiol ${ }^{34}$ ), the biosynthesis of which (encoded by $m s h A B C$ gene cluster) was

148 also encoded in Binatota genomes (Figure 3a). Second, the tetrahydrofolate $\left(\mathrm{H}_{4} \mathrm{~F}\right)-$ linked

149 pathway comprising the genes folD (encoding bifunctional methylene- $\mathrm{H}_{4} \mathrm{~F}$ dehydrogenase and

150 methenyl- $\mathrm{H}_{4} \mathrm{~F}$ cyclohydrolase) and either $f t f L$ (the reversible formyl- $\mathrm{H}_{4} \mathrm{~F}$ ligase) or $\operatorname{pur} U$ (the

151 irreversible formyl-H4F hydrolase) was also widespread (98/108 genomes). Finally, 40 genomes

152 (Bin18, Binatales, HRBin30, and UTPR01) also encoded the single gene/enzyme NAD-linked

153 glutathione-independent formaldehyde dehydrogenase $f d h A$. Surprisingly, no evidence of the

154 most common formaldehyde oxidation pathway (tetrahydromethanopterin ( $\left.\mathrm{H}_{4} \mathrm{MPT}\right)$-linked) was

155 detected in any of the Binatota genomes. The NAD- and glutathione-dependent formaldehyde 
156 oxidation pathway was found incomplete: while homologs of formaldehyde dehydrogenase

$157($ frm $A)$ were detected in almost all Binatota genomes, S-formylglutathione hydrolase $($ frm $B)$ were

158 absent. Following formaldehyde oxidation to formate, formate is subsequently oxidized to $\mathrm{CO}_{2}$

159 by one of many formate dehydrogenases. The majority of Binatota genomes (103/108) encoded

160 at least one copy of the NAD-dependent formate dehydrogenase (EC: 1.17.1.9) (Figure 3a,

161 Extended data 1).

162 3. Formaldehyde assimilation. Two pathways for formaldehyde assimilation by methylotrophs

163 have been described: the serine cycle, which assimilates 2 formaldehyde molecules and $1 \mathrm{CO}_{2}$

164 molecule, and the ribulose monophosphate cycle (RuMP), which assimilates 3 formaldehyde and

165 no $\mathrm{CO}_{2}$ molecules. In addition, some methylotrophs assimilate carbon at the level of $\mathrm{CO}_{2}$ via the

166 Calvin Benson Bassham (CBB) cycle ${ }^{22}$. Homologs encoding the RuMP cycle-specific enzymes

167 were missing from all Binatota genomes, and only three genomes belonging to the Binatales

168 order encoded the CBB cycle enzymes phosphoribulokinase and rubisCO. On the other hand,

169 genes encoding enzymes of the serine cycle (Figure 3b) were identified in all genomes (Figure

$1703 \mathrm{c}$, Extended data 1), with the key enzymes that synthesize and cleave malyl-CoA (mtkA/B $[\mathrm{EC}$

171 6.2.1.9] malate-CoA ligase, and $m c l$ [EC 4.1.3.24] malyl-CoA lyase, respectively) encountered

172 in 98, and 86 Binatota genomes, respectively (Figure 3c, Extended data 1). The entry point of

$173 \mathrm{CO}_{2}$ to the serine cycle is the phosphoenolpyruvate (PEP) carboxylase ( $\left.p p c\right)$ step catalyzing the

174 carboxylation of PEP to oxaloacetate (Figure 3b). Homologues of $p p c$ were missing from most

175 Binatota genomes. Instead, all genomes encoded PEP carboxykinase $(p c k A)$ that replaces $p p c$

176 function as shown in methylotrophic mycobacteria ${ }^{35}$ (Figure 3b-c, Extended data 1).

177 During the serine cycle, regeneration of glyoxylate from acetyl-CoA is needed to restore

178 glycine and close the cycle. Glyoxylate regeneration can be realized either through the classic 
179 glyoxylate shunt ${ }^{36}$, or the ethylmalonyl-CoA pathway (EMCP) ${ }^{37}$ (Figure 3b). All Binatota

180 genomes exhibited the capacity for glyoxylate regeneration, but the pathway employed appears

181 to be order-specific. Genes encoding all EMCP pathway enzymes were identified in genomes

182 belonging to the orders Bin18, Binatales, HRBin30, UBA1149, and UBA12105 (Figure 3c,

183 Extended data 1), including the two EMCP-specific enzymes ethylmalonyl-CoA mutase (ecm)

184 and crotonyl-CoA reductase/carboxylase (ccr). On the other hand, order UBA9968 genomes

185 lacked EMCP-specific enzymes but encoded the classic glyoxylate shunt enzymes isocitrate

186 lyase $(a c e A)$ and malate synthase $(a c e B)$ (Figure 3c, Extended data 1).

\section{Alkane degradation}

188 Besides methylotrophy and methanotrophy, Binatota genomes exhibited extensive short-,

189 medium-, and long-chain alkanes degradation capabilities. In addition to the putative capacity of

190 Actinobacteria/SAR324-affiliated pMMO to oxidize $\mathrm{C}_{1}-\mathrm{C}_{5}$ alkanes, and $\mathrm{C}_{1}-\mathrm{C}_{4}$ alkenes as

191 described above, some Binatota genomes encoded propane-2-monoxygenase (prmABC), an

192 enzyme mediating propane hydroxylation in the 2-position yielding isopropanol. Several

193 genomes, also encoded medium chain-specific alkane hydroxylases, e.g. homologues of the

194 nonheme iron $a l k B^{38}$ and Cyp153-class alkane hydroxylases ${ }^{39}$. The genomes also encoded

195 multiple long-chain specific alkane monooxygenase, e.g. ladA homologues (EC:1.14.14.28) ${ }^{40}$

196 (Figure 4a, Extended data 1). Finally, Binatota genomes encoded the capacity to metabolize

197 medium-chain haloalkane substrates. All genomes encoded dhaA (haloalkane dehalogenases

198 [EC:3.8.1.5]) known to have a broad substrate specificity for medium chain length (C3 to C10)

199 mono-, and dihaloalkanes, resulting in the production of their corresponding primary alcohol,

200 and haloalcohols, respectively ${ }^{41}$ (Figure 4a, Extended data 1). 
Alcohol and aldehyde dehydrogenases sequentially oxidize the resulting alcohols to their corresponding fatty acids or fatty acyl-CoA. Binatota genomes encode a plethora of alcohol and

203 aldehyde dehydrogenases. These include the wide substrate range alcohol (EC:1.1.1.1), and

204 aldehyde (EC:1.2.1.3) dehydrogenases encoded by the majority of Binatota genomes, as well as

205 bifunctional alcohol/aldehyde dehydrogenase (EC:1.2.1.10/1.1.1.1) encoded by a few Binatota

206 genomes (7 genomes), and some highly specific enzymes, e.g. the short-chain isopropanol

207 dehydrogenase (EC:1.1.1.80) for converting isopropanol and other secondary alcohols to the

208 corresponding ketone (20 genomes), and acetone monooxygenase ( $\mathrm{acm} A$, EC:1.14.13.226) and

209 methyl acetate hydrolase $(a c m B, E C: 3.1 .1 .114)$ that will sequentially oxidize acetone to

210 methanol and acetate (6 genomes) (Figure 4a, Extended data 1).

211 A Complete fatty acid degradation machinery that enables all orders of the Binatota to

212 degrade short-, medium-, and long-chain fatty acids to acetyl CoA and propionyl-CoA were

213 identified (Figure 4b, Extended data 1). Acetyl-CoA produced from the beta-oxidation pathway

214 could be assimilated via the ethylmalonyl CoA pathway (EMCP) or the glyoxylate shunt as

215 discussed above. Further, two pathways for propionyl-CoA assimilation, generated from the

216 degradation of odd chain fatty acids, were identified (Figure 4c). Orders Bin18, Binatales,

217 UBA1149, UBA12105, and UTPR01 all encode enzymes for the methylmalonyl CoA (MMCoA)

218 pathway that carboxylates propionyl CoA to succinyl-CoA (TCA cycle intermediate) via a

219 methylmalonyl-CoA intermediate. On the other hand, the majority of order UBA9968 genomes

220 encode enzymes of the 2-methylcitrate cycle for propionyl-CoA degradation ( $p r p B C D$ ) where

221 propionate is degraded to pyruvate and succinate via a 2-methylcitrate intermediate (Figures 4b-

222 c, Extended data 1).

\section{Electron transport chain}


224 All Binatota genomes encode an aerobic respiratory chain comprising complexes I, II, and IV, as

225 well as an F-type $\mathrm{H}^{+}$-translocating ATP synthase (Figures 5a-b, Extended data 1). Interestingly,

226 genes encoding complex III (cytochrome bc1 complex) were sparse in Binatota genomes with

227 some orders lacking genes encoding all subunits (e.g. HRBin30) and others only encoding the

228 Fe-S (ISP) and the cytochrome $\mathrm{b}(c y t B)$ but not the cytochrome c1 (cytl) subunit (e.g. Binatales,

229 UBA1149). Instead, genes encoding an Alternate Complex III (ACIII, encoded by

230 act $A B C D E F G$ ) were identified in 76 genomes, with 12 genomes encoding both complete

231 complexes (in orders Bin 18, UBA9968, and UTPR01). Complex III and ACIII transfer electrons

232 from reduced quinones (all genomes encode the capability of menaquinone biosynthesis) to

233 cytochrome $\mathrm{c}$ which, in turn, reduces cytochrome c oxidase (complex IV). Homologues of the

234 electron transfer proteins belonging to cytochrome c families were rare in Binatota genomes,

235 especially those encoding ACIII (Figure 5a, Extended data 1). However, the recent structure of

236 ACIII from Flavobacterium johnsoniae ${ }^{42}$ in a supercomplex with cytochrome c oxidase aa3

237 suggests that electrons could potentially flow from ACIII to complex IV without the need for

238 cytochrome c, which might explain the paucity of cytochrome c homologues in ACIII-harboring

239 genomes.

Based on the predicted ETC structure, the flow of electrons under different growth

241 conditions in the Binatota could be envisaged (Figure 5b). When growing on methane, pMMO

242 would be coupled to the electron transport chain at complex III level via the quinone pool, where

243 reduced quinones would act as physiological reductant of the enzyme ${ }^{43}$ (Figure 5b). pMMO was

244 also previously reported to receive electrons donated by $\mathrm{NADH}^{44}$. During methanol oxidation by

245 periplasmic enzymes (e.g. xoxF-type methanol dehydrogenases), and methylamine oxidation by

246 the periplasmic methylamine dehydrogenase $(\operatorname{mau} A B)$ electrons would be shuttled via their 
247 respective C-type cytochrome (xoxG, and mauC, respectively) to complex IV. In the cytosol,

248 methanol oxidation via the $m n o / m d o$-type or the $m d h 2$-type methanol dehydrogenases, as well as

249 formaldehyde and formate oxidation via the action of cytoplasmic formaldehyde and formate

250 dehydrogenases would contribute NADH to the aerobic respiratory chain through complex I.

251 Similarly, when growing heterotrophically on alkanes and/or fatty acids, reducing equivalents in

252 the form of $\mathrm{NAD}(\mathrm{P}) \mathrm{H}$, and $\mathrm{FADH}_{2}$ serve as electron donors for aerobic respiration through

253 complex I, and II, respectively (Figure 5b).

254 Binatota genomes also encode respiratory $\mathrm{O}_{2}$-tolerant $\mathrm{H}_{2}$-uptake $[\mathrm{NiFe}]$ hydrogenases,

255 belonging to groups 1c (6 sequences), 1f (22 sequences), 1i (1 sequence), and 1h (4 sequences)

256 (Figure 5c). In E. coli, these membrane-bound periplasmically oriented hydrogenases transfer

257 electrons (through their cytochrome b subunit) from molecular hydrogen to the quinone pool.

258 Cytochrome $b d$ oxidase (complex IV) then completes this short respiratory electron transport

259 chain between $\mathrm{H}_{2}$ and $\mathrm{O}_{2}{ }^{45}$. In $E$. coli, the enzyme functions under anaerobic conditions ${ }^{46}$, and

260 may function as an $\mathrm{O}_{2}$-protecting mechanism ${ }^{47}$. Further, simultaneous oxidation of hydrogen

261 (via type I respiratory $\mathrm{O}_{2}$-tolerant hydrogenases) and methane (via pMMO) has been shown to

262 occur in methanotrophic Verrucomicrobia to maximize proton-motive force generation and

263 subsequent ATP production ${ }^{48}$. As well, some of the reduced quinones generated through $\mathrm{H}_{2}$

264 oxidation are thought to provide reducing power for catalysis by pMMO $^{48}$ (Figure $5 b$ ).

265 Pigment production genes in the Binatota.

266 Carotenoids. Analysis of the Binatota genomes demonstrated a wide range of hydrocarbon

267 (carotenes) and oxygenated (xanthophyll) carotenoid biosynthesis capabilities. Carotenoids

268 biosynthetic machinery in the Binatota included $\operatorname{crt} B$ for 15-cis-phyotene synthesis from

269 geranylgeranyl-PP; $c r t I, c r t P, c r t Q$, and $c r t H$ for neurosporene and all-trans lycopene formation 
270 from 15-cis-phytone; $\operatorname{crt} Y$ or $\operatorname{crtL}$ for gamma- and beta-carotene formation from all-trans

271 lycopene; and a wide range of genes encoding enzymes for the conversion of neurosporene to

272 spheroidene and 7,8-dihydro $\beta$-carotene, as well as the conversion of all-trans lycopene to

273 spirilloxanthin, gamma-carotene to hydroxy-chlorobactene glucoside ester and hydroxy-V-

274 carotene glucoside ester, and beta carotene to isorenieratene and zeaxanthins (Figures 6a-b,

275 Extended data 1). Gene distribution pattern (Figure 6a, Extended data 1) predicts that all Binatota

276 orders are capable of neurosporene and all-trans lycopene biosynthesis, and all but the order

277 HRBin30 are capable of isorenieratene, zeaxanthin, $\beta$-carotene and dihydro $\beta$-carotene

278 biosynthesis, and with specialization of order UTPR01 in spirilloxanthin, spheroidene, hydroxy-

279 chlorobactene, and hydroxy Y-carotene biosynthesis.

280 Bacteriochlorophylls. Surprisingly, homologues of multiple genes involved in

281 bacteriochlorophyll biosynthesis were ubiquitous in Binatota genomes (Figure 7a-c).

282 Bacteriochlorophyll biosynthesis starts with the formation of chlorophyllide $a$ from

283 protoporphyrin IX (Figure 7b). Within this pathway, genes encoding the first bchI (Mg-chelatase

284 [EC:6.6.1.1]), third $b c h E$ (magnesium-protoporphyrin IX monomethyl ester cyclase

285 [EC:1.21.98.3]), and fourth $b \operatorname{ch} L N B$ (3,8-divinyl protochlorophyllide reductase [EC:1.3.7.7])

286 steps were identified in the Binatota genomes (Figures 7a, 7b, Extended data 1). However,

287 homologues of genes encoding the second bchM (magnesium-protoporphyrin O-

288 methyltransferase [EC:2.1.1.11]), and the fifth (bciA or bicB (3,8-divinyl protochlorophyllide $a$

289 8-vinyl-reductase), or $b \operatorname{ch} X Y Z$ (chlorophyllide $a$ reductase, EC 1.3.7.15])) steps were absent

290 (Figure 7a-b). A similar patchy distribution was observed in the pathway for bacteriochlorophyll

$291 a(\mathrm{Bchl} a)$ formation from chlorophyllide $a$ (Figure $7 \mathrm{~b})$, where genes encoding $b c h X Y Z$

292 (chlorophyllide $a$ reductase [EC 1.3.7.15]) and $b c h F$ (chlorophyllide $a 3^{1}$-hydratase [EC 
4.2.1.165]) were not identified, while genes encoding $b c h C$ (bacteriochlorophyllide $a$ dehydrogenase [EC 1.1.1.396]), bch $G$ (bacteriochlorophyll a synthase [EC:2.5.1.133]), and $b c h P$

295 (geranylgeranyl-bacteriochlorophyllide $a$ reductase [EC 1.3.1.111)) were present in most

296 genomes (Figure 7a, Extended data 1). Finally, within the pathway for bacteriochlorophylls $c$

297 (Bchl $c)$ and $d(\mathrm{Bchl} d)$ formation from chlorophyllide $a$ (Figure $7 \mathrm{~b}$ ), genes for $b c i C$

298 (chlorophyllide a hydrolase [EC:3.1.1.100]), and $b c h F$ (chlorophyllide $a 3^{1}$-hydratase

299 [EC:4.2.1.165]) or bchV (3-vinyl bacteriochlorophyllide hydratase [EC:4.2.1.169] were not

300 identified, while genes for $b \operatorname{ch} R$ (bacteriochlorophyllide d C-12(1)-methyltransferase

301 [EC:2.1.1.331]), bchQ (bacteriochlorophyllide d C-8(2)-methyltransferase [EC:2.1.1.332]), bchU

302 (bacteriochlorophyllide d C-20 methyltransferase [EC:2.1.1.333]), and $b c h K$

303 (bacteriochlorophyll c synthase [EC:2.5.1.-]) were identified (Figure 7b, Extended data 1).

\section{Ecological distribution of the Binatota.}

305 A total of 1,889 (GenBank nt) and 1,213 (IMG/M) 16S rRNA genes affiliated with the Binatota 306 orders were identified (Extended data 2 and 3, Figures 8, S1a). Analyzing their environmental 307 distribution showed preference of Binatota to terrestrial soil habitats $(39.5-83.0 \%$ of GenBank, $308 \quad 31.7-91.6 \%$ of IMG/M 16S rRNA gene sequences in various orders), as well as plant-associated

309 (particularly rhizosphere) environments; although this could partly be attributed to sampling bias 310 of these globally distributed and immensely important ecosystems (Figure 8a). On the other

311 hand, a paucity of Binatota-affiliated sequences was observed in marine settings, with sequences

312 absent or minimally present for Binatales, HRBin30, UBA9968, and UTPRO1 datasets (Figure

313 8a). The majority of sequences from marine origin were sediment-associated, being encountered

314 in hydrothermal vents, deep marine sediments, and coastal sediments, with only the Bin18 
315 sequences sampled from IMG/M showing representation in the vast, relatively well-sampled

316 pelagic waters (Figure 8d).

317 In addition to phylum-wide patterns, order-specific environmental preferences were also

318 observed. For example, in order Bin18, one of the two available genomes originated from the

319 Mediterranean sponge Aplysina aerophoba. Analysis of the 16S rRNA dataset suggests a notable

320 association between Bin18 and sponges, with a relatively high host-associated sequences (Figure

321 8a), the majority of which (58.3\% NCBI-nt, 25.0\% IMG/M) were recovered from the Porifera

322 microbiome (Figures 8e, S1f). Bin18-affiliated 16S rRNA gene sequences were identified in a

323 wide range of sponges from ten genera and five global habitat ranges (the Mediterranean genera

324 Ircinia, Petrosia, Chondrosia, and Aplysina, the Caribbean genera Agelas, Xestospongia, and

325 Aaptos, the Indo-West Pacific genus Theonella, the Pacific Dysideidae family, and the Great

326 Barrier Reef genus Rhopaloeides), suggesting its widespread distribution beyond a single sponge

327 species. The absolute majority of order Binatales sequences (83.0\% NCBI-nt, 91.6\% IMG/M)

328 were of a terrestrial origin (Figures 8a, S1c), in addition to multiple rhizosphere-associated

329 samples (7.5\% NCBI-nt and 2.8\% IMG/M, respectively) (Figure 8a, S1f). Notably, a relatively

330 large proportion of Binatales soil sequences originated either from wetlands (peats, bogs) or

331 forest soils (Figures 8b, S1c), strongly suggesting the preference of the order Binatales to acidic

332 and organic/methane-rich terrestrial habitats. This corresponds with the fact that 42 out of 48

333 Binatales genomes were recovered from soil, 38 of which were from acidic wetland or forest

334 soils (Figure 1, Table S1). Genomes of UBA9968 were recovered from a wide range of

335 terrestrial and non-marine aquatic environments, and the observed 16S rRNA gene distribution

336 enforces their ubiquity in all but marine habitats (Figures 8a, S1b-g). Finally, while genomes

337 from orders HRBin30, UBA1149 and UTPRO1 were recovered from limited environmental 
338 settings (thermal springs for HRBin30, gaseous hydrocarbon impacted habitats, e.g. marine

339 hydrothermal vents and gas-saturated Lake Kivu for UBA1149, and soil and hydrothermal

340 environments for UTPRO1) (Figure 1, Table S1), 16S rRNA gene analysis suggested their

341 presence in a wide range of environments from each macro-scale environment classification

342 (Figures 8a, S1b-g). 


\section{Discussion}

344 Expanding the world of methylotrophy. The current study expands the list of lineages

345 potentially capable of methylotrophy. An extensive repertoire of genes and pathways mediating

346 the oxidation of multiple $\mathrm{C} 1$ compounds to formaldehyde (Figure 2, 9), formaldehyde oxidation

347 to $\mathrm{CO}_{2}$ (Figure 3a), as well as formaldehyde assimilation pathways (Figure 3c) were identified,

348 indicating that such capacity is a defining metabolic trait in the Binatota. A certain degree of

349 order-level substrate preference was observed, with potential utilization of methanol in all orders

350 except HRBin30, methylamine in all orders except UBA9968, S-containing C1 compound in

351 Bin18, Binatales, and UBA9968, halogenated methane in Bin18, and possible methane

352 utilization (methanotrophy) in Bin18 and Binatales (Figure 2a).

353 Aerobic methylotrophy has been documented in members of the alpha, beta, and gamma

354 Proteobacteria ${ }^{49}$, Bacteroidetes ${ }^{50}$, Actinobacteria (e.g. genera Arthrobacter and

355 Mycobacterium), Firmicutes (e.g. Bacillus methanolicus) ${ }^{51}$, Verrucomicrobia ${ }^{52}$, and Candidatus

356 Methylomirabilis (NC10) ${ }^{53}$. Further, studies employing genome-resolved metagenomics

357 identified some signatures of methylotrophy, e.g. methanol oxidation ${ }^{10,54}$, formaldehyde

358 oxidation/ assimilation ${ }^{55}$, and methylamine oxidation ${ }^{10}$, in the Gemmatimonadetes,

359 Rokubacteria, Chloroflexi, Actinobacteria, Acidobacteria, and Lambdaproteobacteria. The

360 possible contribution of Binatota to methane oxidation (methanotrophy) is especially notable,

361 given the global magnitude of methane emissions ${ }^{56}$, and the relatively narrower range of

362 organisms (Proteobacteria, Verrucomicrobia, and Candidatus Methylomirabilota (NC10)) ${ }^{57}$

363 capable of this special type of methylotrophy. As described above, indirect evidence exists for

364 the involvement of Binatota harboring TUSC-type pMMO sequences in methane oxidation,

365 while it is currently uncertain whether Binatota harboring SAR324/Actinobacteria-type pMMO 
sequences are involved in oxidation of methane, gaseous alkanes, or both. pMMO of

methanotrophs is also capable of oxidizing ammonia to hydroxylamine, which necessitates genomes encoding pMMO also encoded at least one homologue of nir, nor, and/or nos genes that could potentially convert harmful N-oxide byproducts to dinitrogen. assimilation. Within the world of methylotrophs, a wide array of functionally redundant enzymes/pathways has been characterized that mediates various reactions/ transformations in such modules. In addition, multiple combinations of different modules have been observed in methylotrophs, with significant variations existing even in phylogenetically related organisms.

377 Our analysis demonstrates that such metabolic versatility indeed occurs within Binatota's

378 methylotrophic modules. While few phylum-wide characteristics emerged, e.g. utilization of

379 serine pathway for formaldehyde assimilation, absence of $\mathrm{H}_{4} \mathrm{MPT}$-linked formaldehyde

380 oxidation, and potential utilization of PEP carboxykinase ( $p c k A)$ rather than PEP carboxylase

381 ( $p p c)$ for $\mathrm{CO}_{2}$ entry to the serine cycle, multiple order-specific differences were observed, e.g.

382 XoxF-type methanol dehydrogenase encoded by Bin18 and Binatales genomes, MDH2-type

383 methanol dehydrogenase encoded by UBA1149 genomes, absence of methanol dehydrogenase

384 homologues in HRBin30 genomes, absence of methylamine oxidation in order UBA9968, and

385 potential utilization of the ethylmalonyl-CoA pathway for glyoxylate regeneration by the

386 majority of the orders versus the glyoxylate shunt by UBA9968.

387 Alkane degradation in the Binatota. A second defining feature of the phylum Binatota, besides

388 methylotrophy, is the widespread capacity for aerobic alkane degradation, as evident by the 
extensive arsenal of genes mediating aerobic degradation of short- (pMMO, propane

monooxygenase), medium- (alkB, cyp153), and long-chain alkanes (ladA) identified (Figure 4a),

391 in addition to complete pathways for odd- and even-numbered fatty acids oxidation (Figure 4b).

392 Hydrocarbons, including alkanes, have been an integral part of the earth biosphere for eons, and

393 a fraction of microorganisms has evolved specific mechanisms $\left(\mathrm{O}_{2}\right.$-dependent hydroxylases and

394 monooxygenases, anaerobic addition of fumarate) for their activation and conversion to central

395 metabolites ${ }^{59}$. Aerobic alkane degradation capacity has so far been encountered in the

396 Actinobacteria, Proteobacteria, Firmicutes, Bacteroidetes, as well as in a few Cyanobacteria ${ }^{59}$.

397 As such, this study adds to the expanding list of phyla capable of aerobic alkane degradation.

398 Metabolic traits explaining niche preferences in the Binatota. Analysis of 16S rRNA gene

399 datasets indicated that the Binatota display phylum-wide (preference to terrestrial habitats and

400 methane/hydrocarbon-impacted habitats, and rarity in pelagic marine environments), as well as

401 order-specific (Bin18 in sponges, HRBin30 and UBA1149 in geothermal settings, Binatales in

402 peats, bogs, and forest soils) habitat preferences (Figures 8, S1). Such distribution patterns could

403 best be understood in light of the phylum's predicted metabolic capabilities. Soils represent an

404 important source of methane, generated through microoxic and anoxic niches within soil's

405 complex architecture ${ }^{60}$. Methane emission from soil is especially prevalent in peatlands, bogs,

406 and wetlands, where incomplete aeration and net carbon deposition occurs. Indeed, anaerobic ${ }^{61}$,

407 fluctuating ${ }^{62}$, and even oxic ${ }^{63}$ wetlands represent one of the largest sources of methane

408 emissions to the atmosphere. As well, terrestrial ecosystems represent a major source of global

409 methanol emissions ${ }^{64}$, with its release mostly mediated by demethylation reactions associated

410 with pectin and other plant polysaccharides degradation. C1-metabolizing microorganisms

411 significantly mitigate methane and methanol release to the atmosphere from terrestrial 
412 ecosystems ${ }^{65}$, and we posit that members of the Binatota identified in soils, rhizosphere, and

413 wetlands contribute to such process. The special preference of order Binatales to acidic peats,

414 bogs, forests, and wetlands could reflect a moderate acidophilic specialization for this order and

415 suggest their contribution to the process in these habitats.

416 Within the phylum Binatota, it appears that orders HRBin30 and UBA1149 are abundant

417 in thermal vents, thermal springs, and thermal soils, suggesting a specialization to high

418 temperature habitats (Figure 8). Binatota's presence in such habitats could be attributed to high

419 concentrations of alkanes typically encountered in such habitats. Hydrothermal vents display

420 steep gradients of oxygen in their vicinity, emission of high levels of methane and other gaseous

421 alkanes, as well as thermogenic generation of medium-and long-chain alkanes ${ }^{66}$. Indeed, the

422 presence and activity of aerobic hydrocarbon degraders in the vicinity of hydrothermal vents

423 have been well established $30,31,67$.

424 The recovery of Binatota genomes from certain lakes could be a reflection of the high

425 gaseous load in such lakes. Multiple genomes and a large number of Binatota-affiliated 16S

426 rRNA sequences were binned/identified from Lake Kivu, a meromictic lake characterized by

427 unusually high concentrations of methane ${ }^{68}$. Biotically, methane evolving from Lake Kivu is

428 primarily oxidized by aerobic methanotrophs in surface waters ${ }^{68,69,70}$, and members of the

429 Binatota could contribute to this process. Binatota genomes were also recovered from Lake

430 Washington sediments, a location that has long served as a model for studying methylotrophy ${ }^{71,}$

$431 \quad 72$. Steep counter gradients of methane and oxygen occurring in the Lake's sediments enable

432 aerobic methanotrophy to play a major role in controlling methane flux through the water

433 column ${ }^{73,74,75,76 .}$ 

sponges, particularly by the order Bin18, is notable, and could be attributed to the recognized

436 nutritional-based symbiosis between sponges and hydrocarbon-degraders ${ }^{77,78}$, including

437 methanotrophs ${ }^{79}$. This is especially true in deep-water sponges, where low levels of planktonic

438 biomass restrict the amount of food readily acquired via filter feeding and hence biomass

439 acquisition via methane and alkane oxidation is especially valuable.

440 Carotenoid pigmentation: occurrence and significance. The third defining feature of the

441 Binatota, in addition to aerobic methylotrophy and alkane degradation, is the predicted capacity

442 for carotenoid production. In photosynthetic organisms, carotenoids increase the efficiency of

443 photosynthesis by absorbing in the blue-green region then transferring the absorbed energy to the

444 light-harvesting pigments ${ }^{80}$. Carotenoid production also occurs in a wide range of non-

445 photosynthetic bacteria belonging to the Alpha-, Beta, and Gamma-Proteobacteria (including

446 methano- and methylotrophs ${ }^{81}$ ), Bacteroidetes ${ }^{82}$, Deinococcus ${ }^{83}$, Thermus ${ }^{84}$, Delta-

447 Proteobacteria ${ }^{85}$, Firmicutes ${ }^{86}$, Actinobacteria ${ }^{87}$, Planctomycetes ${ }^{88}$, and Archaea, e.g.

448 Halobacteriaceae ${ }^{89}$, and Sulfolobus ${ }^{90}$. Here, carotenoids could serve as antioxidants ${ }^{91}$, and aid

449 in radiation, $\mathrm{UV}$, and desiccation resistance ${ }^{92,93}$. The link between carotenoid pigmentation and

450 methylo/methanotrophy has long been observed ${ }^{94}$, with the majority of known model Alpha-

451 and Gamma-Proteobacteria methano- and methylotrophs being carotenoid producers, although

452 several Gram-positive methylotrophs (Mycobacterium, Arthrobacter, and Bacillus) are not

453 pigmented. Indeed, root-associated facultative methylotrophs of the genus Methylobacterium

454 have traditionally been referred to as "pink pigmented facultative methylotrophs" and are seen as

455 integral part of root ecosystems ${ }^{95}$. The exact reason for this correlation is currently unclear and

456 could be related to the soil environment where they are prevalent, where periodic dryness and 
457 desiccation could occur, or to the continuous exposure of these aerobes in some habitats to light

458 (e.g. in shallow sediments), necessitating protection from UV exposure.

459 Chlorophyll biosynthesis genes in the Binatota. Perhaps the most intriguing finding in this

460 study is the identification of the majority of genes required for the biosynthesis of

461 bacteriochlorophylls from protoporphyrin-IX (six out of ten genes for bacteriochlorophyll $a$ and

462 seven out of eleven genes for bacteriochlorophyll $c$ and $d$ ). While such pattern is tempting to

463 propose phototrophic capacities in the Binatota based on the common practice of using a certain

464 percentage completion threshold to denote pathway occurrence in some studies (e.g. ${ }^{2}$ ), the

465 consistent absence of critical genes (bchM methyltransferase, bciA/bciB/bchXYZ reductases, bciC

466 hydrolase, and $b c h F / V$ hydratases), coupled with our inability to detect reaction center-encoding

467 genes, prevents such a proclamation. Identification of a single or few gene shrapnel from the

468 chlorophyll biosynthesis pathway in microbial genomes is not unique. Indeed, searching the

469 functionally annotated bacterial tree of life AnnoTree ${ }^{96}$ using single KEGG orthologies

470 implicated in chlorophyll biosynthesis identifies multiple (in some cases thousands) hits in

471 genomes from non-photosynthetic organisms (Figure 7c). This is consistent with the

472 identification of a $b c h G$ gene in a Bathyarchaeota fosmid clone ${ }^{97}$, and, more recently, a few

473 bacteriochlorophyll synthesis genes in an Asgard genome ${ }^{98}$. However, it should be noted that the

474 high proportion of genes in the bacteriochlorophyll biosynthetic pathway identified in the

475 Binatota genomes has never previously been encountered in non-photosynthetic microbial

476 genomes. Indeed, a search in AnnoTree for the combined occurrence of all seven

477 bacteriochlorophyll synthesis genes identified in Binatota genomes yielded only photosynthetic

478 organisms. 
Accordingly, we put forward three scenarios to explain the proposed relationship between Binatota and phototrophy: The most plausible scenario, in our opinion, is that members of the Binatota are pigmented non-photosynthetic organisms capable of carotenoid production, but incapable of chlorophyll production and lack a photosynthetic reaction center. Under this scenario, the incomplete pathway for bacteriochlorophyll biosynthesis represents a pattern of

484 gene loss from a chlorophyll-producing ancestor. The assumption that a lineage has lost the immensely beneficial capacity to harvest energy from light might appear counterintuitive, even implausible. However, this could be understood in the context of the proposed role of chlorophyll during the early evolution of photosynthesis. In a thought-provoking review, Martin et al. ${ }^{99}$ argue that the evolution of chlorophyll-based biosynthesis occurred against a backdrop of

489 chemolithotrophy in hydrothermal vents, with hydrogen produced abundantly by serpentinization 490 as the main source of energy, and $\mathrm{CO}_{2}$ fixation via the acetyl $\mathrm{CoA}$ pathway as the main source of 491 carbon. The acetyl-CoA pathway requires electron transfer to an acceptor, ferrodoxin, with an 492 extremely negative midpoint potential, which could only be achieved via electron bifurcation 493 reactions. Within such chemolithotrophic, dim-lit hydrogen-dominated realm, the main benefit of 494 chlorophyll-based anoxygenic photosynthesis would be harvesting the relatively limited amount 495 of thermal light emitted from hydrothermal vents ${ }^{100}$ to allow access to a new source of 496 moderately low-potential electrons $\left(\mathrm{H}_{2} \mathrm{~S}\right.$ as opposed to $\left.\mathrm{H}_{2}\right)$ that could be used together with light 497 energy to generate reduced ferredoxin for the purpose of $\mathrm{CO}_{2}$ fixation via the ferredoxin498 dependent acetyl-CoA pathway. The need for such function in a microorganism would be 499 alleviated with the development of heterotrophic capacities and acquisition of additional 500 pathways for energy production, allowing for the loss of the non-utilized chlorophyll synthesis 501 pathway. 
The second scenario posits that members of the Binatota are indeed phototrophs,

503 possessing a complete pathway for chlorophyll biosynthesis and a novel type of reaction center

504 that is bioinformatically unrecognizable. A minimal photosynthetic electron transport chain,

505 similar to Chloroflexus aurantiacus ${ }^{101}$, with the yet-unidentified reaction center, quinone,

506 alternate complex III (or complex III) and some type of cytochrome c would possibly be

507 functional. Under such scenario, members of the Binatota would be an extremely versatile

508 photoheterotrophic facultative methylotrophic lineage. While such versatility, especially

509 coupling methylotrophy to phototrophy, is rare ${ }^{102}$, it has previously been observed in some

510 Rhodospirillaceae species ${ }^{103}$. A third scenario is that Binatota are capable of chlorophyll

511 production, but still incapable of conducting photosynthesis. Under this scenario, genes missed

512 in the pathway are due to shortcomings associated with in-silico prediction and conservative

513 gene annotation. For example, the missing bchM (E.C.2.1.1.11) could possibly be encoded for by

514 general methyltransferases (EC: 2.1.1.-), the missing bciC (EC:3.1.1.100) could possibly be

515 encoded for by general hydrolases (EC: 3.1.1.-), while the missing $b c h F$ (EC:4.2.1.165) or $b c h V$

516 (EC:4.2.1.169) could possibly be encoded for by general hydratases (EC: 4.2.1.-).

517 Encountering incomplete pathways in genomes of uncultured lineages is an exceedingly

518 common occurrence in SAG and MAG analysis ${ }^{104,105}$. In many cases, this could plausibly

519 indicate an incomplete contribution to a specific biogeochemical process, e.g. incomplete

520 denitrification of nitrate to nitrite but not ammonia ${ }^{105}$, or reduction of sulfite, but not sulfate, to

521 sulfide ${ }^{106}$, provided the thermodynamic feasibility of the proposed partial pathway, and,

522 preferably, prior precedence in pure cultures. In other cases, a pattern of absence of peripheral

523 steps could demonstrate the capability for synthesis of a common precursor, e.g., synthesis of

524 precorrin-2 from uroporphyrinogen, but lack of the peripheral pathway for corrin ring 
525 biosynthesis leading to an auxotrophy for vitamin B12. Such auxotrophies are common in the

526 microbial world and could be alleviated by nutrient uptake from the outside environment ${ }^{107}$ or

527 engagement in a symbiotic lifestyle ${ }^{108}$. However, arguments for metabolic interdependencies,

528 syntrophy, or auxotrophy could not be invoked to explain the consistent absence of specific

529 genes in a dedicated pathway, such as bacteriochlorophyll biosynthesis, especially when

530 analyzing a large number of genomes from multiple habitats. As such, we here raise awareness

531 that using a certain occurrence threshold to judge a pathway's putative functionality could lead to

532 misinterpretations of organismal metabolic capacities due to the frequent occurrence of partial,

533 non-functional, pathways and "gene shrapnel" in microbial genomes.

534 In conclusion, our work provides a comprehensive assessment of the yet-uncultured

535 phylum Binatota, and highlights its aerobic methylotrophic and alkane degradation capacities, as

536 well as its carotenoid production, and abundance of bacteriochlorophyll synthesis genes in its

537 genomes. We also propose a role for this lineage in mitigating methane and methanol emissions

538 from terrestrial and freshwater ecosystems, alkanes degradation in hydrocarbon-rich habitats, and

539 nutritional symbiosis with marine sponges. We present specific scenarios that could explain the

540 unique pattern of chlorophyll biosynthesis gene occurrence, and stress the importance of detailed

541 analysis of pathways completion patterns for appropriate functional assignments in genomes of

542 uncultured taxa. 
545 Genomes. All genomes classified as belonging to the Binatota in the GTDB database ( $\mathrm{n}=22$

546 MAGs, April 2020) were downloaded as assemblies from NCBI. In addition, 128 metagenome-

547 assembled genomes with the classification "Bacteria;UBP10" were downloaded from the

548 IMG/M database (April 2020). These genomes were recently assembled from public

549 metagenomes as part of a wider effort to generate a genomic catalogue of Earth's microbiome ${ }^{16}$.

550 Finally, 6 metagenome-assembled genomes were obtained as part of the Microbial Dark Matter

551 MDM-II project. CheckM ${ }^{109}$ was utilized for estimation of genome completeness, strain

552 heterogeneity, and genome contamination. Only genomes with $>70 \%$ completion and $<10 \%$

553 contamination $(\mathrm{n}=108)$ were retained for further analysis (Tables S1, S2). MAGs were classified

554 as high-, or medium-quality drafts based on the criteria set forth by ${ }^{18}$.

555 Phylogenetic analysis. Taxonomic classifications followed the Genome Taxonomy Database

556 (GTDB) release r89 ${ }^{14,110}$, and were carried out using the classify_workflow in GTDB-Tk ${ }^{111}$

557 (v1.1.0). Phylogenomic analysis utilized the concatenated alignment of a set of 120 single-copy

558 marker genes ${ }^{14,110}$ generated by the GTDB-Tk. Maximum-likelihood phylogenomic tree was

559 constructed in RAxML ${ }^{112}$ (with a cultured representative of the phylum Deferrisomatota as the

560 outgroup). SSU rRNA gene-based phylogenetic analysis was also conducted using 16S rRNA

561 gene sequences extracted from genomes using RNAmmer ${ }^{113}$. Putative taxonomic ranks were

562 deduced using average amino acid identity (AAI; calculated using AAI calculator [http://enve-

563 omics.ce.gatech.edu/]), with the arbitrary cutoffs $56 \%$, and $68 \%$ for family, and genus,

564 respectively.

565 Annotation. Protein-coding genes in genomic bins were predicted using Prodigal ${ }^{114}$. For initial

566 prediction of function, pangenomes were constructed for each order in the phylum Binatota 
567 separately using PIRATE ${ }^{115}$ with percent identity thresholds of $[40,45,50,55,60,65,70,75$,

568 80, 90], a cd-hit step size of 1, and cd-hit lowest percent id of 90. The longest sequence for each

569 PIRATE-identified allele was chosen as a representative and assembled into a pangenome. These

570 pangenomes were utilized to gain preliminary insights on the metabolic capacities and structural

571 features of different orders. BlastKOALA ${ }^{116}$ was used to assign protein-coding genes in each of

572 the pangenomes constructed to KEGG orthologies (KO), which were subsequently visualized

573 using KEGG mapper ${ }^{117}$. Analysis of specific capabilities and functions of interest was

574 conducted on individual genomic bins by building and scanning hidden markov model (HMM)

575 profiles. All predicted protein-coding genes in individual genomes were searched against

576 custom-built HMM profiles for genes encoding C1, alkanes, and fatty acids metabolism, C1

577 assimilation, [NiFe] hydrogenases, electron transport chain complexes, and carotenoid and

578 chlorophyll biosynthesis. To build the HMM profiles, Uniprot reference sequences for all genes

579 with an assigned KO number were downloaded, aligned using Clustal-omega ${ }^{118}$, and the

580 alignment was used to build an HMM profile using hmmbuild (HMMER 3.1b2). For genes not

581 assigned a KO number (e.g. alternative complex III genes, different classes of cytochrome c

582 family, cytochrome P450 medium-chain alkane hydroxylase cyp153, methanol dehydrogenase

$583 \mathrm{MNO} / \mathrm{MDO}$ family), a representative protein was compared against the KEGG Genes database

584 using Blastp and significant hits (those with e-values $<$ e-80) were downloaded and used to build

585 HMM profiles as explained above. The custom-built HMM profiles were then used to scan the

586 analyzed genomes for significant hits using hmmscan (HMMER 3.1b2) with the option -T 100 to

587 limit the results to only those profiles with an alignment score of at least 100. Further

588 confirmation was achieved through phylogenetic assessment and tree building procedures, in

589 which potential candidates identified by hmmscan were aligned to the reference sequences used 
590 to build the custom HMM profiles using Clustal-omega ${ }^{118}$, followed by maximum likelihood

591 phylogenetic tree construction using FastTree ${ }^{119}$. Only candidates clustering with reference

592 sequences were deemed true hits and were assigned to the corresponding KO.

593 Search for photosynthetic reaction center. Identification of genes involved in chlorophyll

594 biosynthesis in Binatota genomes prompted us to search the genomes for photosynthetic reaction

595 center genes. HMM profiles for Reaction Center Type 1 (RC1; PsaAB), and Reaction Center

596 Type $2\left(\mathrm{RC} 2\right.$; PufLM and $\left.\mathrm{PsbD}_{1} \mathrm{D}_{2}\right)$ were obtained from the pfam database (pfam00223 and

597 pfam00124, respectively). Additionally, HMM profiles were built for PscABCD (Chlorobia-

598 specific), PshA/B (Heliobacteria-specific) ${ }^{120}$, as well as the newly identified Psa-like genes from

599 Chloroflexota ${ }^{121}$. The HMM profiles were used to search Binatota genomes for potential hits

600 using hmmscan. To guard against overlooking a distantly related reaction center, we relaxed our

601 homology criteria (by not including -T or -E options during the hmmscan). An additional search

602 using a structurally-informed reaction center alignment ${ }^{120,122}$ was also performed. The best

603 potential hits were modeled using the SWISS-MODEL homology modeler ${ }^{123}$ to check for

604 veracity. Since the core subunits of Type 1 RC proteins are predicted to have 11 transmembrane

$605 \alpha$-helices ${ }^{124,125}$, while type 2 RC are known to contain five transmembrane helices ${ }^{124,126}$, we

606 also searched for all predicted proteins harboring either 5 or 11 transmembrane domains using

607 TMHMM ${ }^{127}$. All identified 5- or 11-helix-containing protein-coding sequences were searched

608 against GenBank protein nr database to identify and exclude all sequences with a predicted

609 function. All remaining 5- or 11-helix-containing proteins with no predicted function were then

610 submitted to SWISS-MODEL homology modeler using the automated mode to predict

611 homology models. 
612 Classification of [NiFe] hydrogenase sequences. All sequences identified as belonging to the

613 respiratory $\mathrm{O}_{2}$-tolerant $\mathrm{H}_{2}$-uptake $[\mathrm{NiFe}]$ hydrogenase large subunit (HyaA) were classified using

614 the HydDB web tool ${ }^{128}$.

615 Particulate methane monooxygenase 3D model prediction and visualization. SWISS-MODEL

$616{ }^{123}$ was used to construct pairwise sequence alignments of predicted Binatota particulate methane

617 monooxygenase with templates from Methylococcus capsulatus str. Bath (pdb: 3RGB), and for

618 predicting tertiary structure models. Predicted models were superimposed on the template

619 enzyme in PyMol (Version 2.0 Schrödinger, LLC).

620 Ecological distribution of Binatota. We queried 16S rRNA sequence databases using

621 representative $16 \mathrm{~S}$ rRNA gene sequences from six out of the seven Binatota orders (order

622 UBA12015 genome assembly did not contain a 16S rRNA gene). Two databases were searched:

623 1. GenBank nucleotide (nt) database (accessed in July 2020) using a minimum identity threshold

624 of $90 \%, \geq 80 \%$ subject length alignment for near full-length query sequences or $\geq 80 \%$ query

625 length for non-full-length query sequences, and a minimum alignment length of $100 \mathrm{bp}$, and 2.

626 The IMG/M 16S rRNA public assembled metagenomes ${ }^{129}$ using a cutoff e-value of $1 \mathrm{e}^{-10}$,

627 percentage similarity $\geq 90 \%$, and either $\geq 80 \%$ subject length for full-length query sequences or

$628 \geq 80 \%$ query length for non-full-length query sequences. Hits satisfying the above criteria were

629 further trimmed after alignment to the reference sequences from each order using Clustal-omega

630 and inserted into maximum likelihood phylogenetic trees in FastTree (v 2.1.10, default settings).

631 The ecological distribution for each of the Binatota orders was then deduced from the

632 environmental sources of their hits. All environmental sources were classified according to the

633 GOLD ecosystem classification scheme ${ }^{130}$. 
634 Data availability. Genomic bins, predicted proteins, and extended data for Figures 2-7 and for

635 Figures 8 and S1a are available at https://github.com/ChelseaMurphy/Binatota. Maximum

636 likelihood trees (Figure 1 and Figure S1a) can be accessed at:

637 https://itol.embl.de/shared/1WgxEjrQfEYWk. Maximum likelihood trees for chlorophyll

638 biosynthesis genes are available at https://itol.embl.de/shared/34y3BUHcQd7Lh.

640 Acknowledgements. This work has been supported by NSF grants 2016423 (to NHY and MSE),

6411441717 and 1826734 (to RS). We thank Dr. Kevin Redding (Arizona State University) for

642 helpful discussions. Work conducted by the U.S. Department of Energy Joint Genome Institute,

643 a DOE Office of Science User Facility, is supported under Contract No. DE-AC02-05CH11231.

644 J.R.S. is supported by NASA Astrobiology Rock Powered Life and was granted U.S. Forest

645 Service permit \#MLD15053 to conduct field work on Cone Pool and the Little Hot Creek,

646 Mammoth Lakes, California. Thanks to students and participants of the 2014-2016

647 International Geobiology Course for research works on Cone Pool. 


\section{Figure legends:}

649 Figure 1. Phylogenomic relationship between analyzed Binatota genomes. The Maximum

650 Likelihood tree was constructed in RAxML from a concatenated alignment of 120 single-copy

651 marker genes. The tree was rooted using Deferrisoma camini (GCA_000526155.1) as the

652 outgroup (not shown). Orders are shown as colored wedges: UBA9968, pink; HRBin30, tan;

653 Bin18, blue; UBA12105, cyan; UTPR01, purple; UBA1149, orange; and Binatales, green.

654 Within each order, families are delineated by grey borders, and genera are shown as colored

655 squares on the branches. Bootstrap values are shown as purple bubbles for nodes with $\geq 70 \%$

656 support. The tracks around the tree represent (innermost-outermost) GC content (with a heatmap

657 that ranges from 53\% (lightest) to 73\% (darkest)), expected genome size (bar chart), and

658 classification of the ecosystem from which the genome originated. All genomes analyzed in this

659 study were $>70 \%$ complete and $<10 \%$ contaminated. Completion/contamination percentages,

660 and individual genomes assembly size are shown in Tables S2, and S3, respectively.

661 Figure 2. C1 substrate degradation capacities in the Binatota. (A) Heatmap of the distribution of

662 various $\mathrm{C} 1$ oxidation genes in Binatota genomes from different orders. The heatmap colors (as

663 explained in the key) correspond to the percentage of genomes in each order encoding a

664 homologue of the gene in the column header. Pathways involving more than one gene for

665 methylamine and methylated sulfur compounds degradation are shown next to the heatmap. To

666 the right, the per-order predicted $\mathrm{C} 1$ oxidation capacity is shown as a heatmap with the colors

667 corresponding to the percentage of genomes in each order where the full degradation pathway

668 was detected for the substrate in the column header. These include $p m o A B C$ for methane,

$669 x$ xoxJG, $m d h 2$, and/or $m n o$ for methanol, $m a u$ and/or indirect glutamate pathway for

670 methylamine, $s f n G$ and $s s u D$ for dimethylsulfone, $d s o, s f n G$ and $s s u D$, or $d m o A$ for 
671 dimethylsulfide (DMS), ssuD for methane sulfonic acid (MSA), and $\operatorname{dcm} A$ for dichloromethane

672 (DCM). pMMO: particulate methane monooxygenase with $p m o A, p m o B, p m o C$, pmoD denoting

673 subunits A, B, C, and D; XoxF-type (xoxF, xoxJ, xoxG), MDH2-type (mdh2), and MNO/MDO-

674 type (mno) methanol dehydrogenases; direct oxidation methylamine dehydrogenase (mauABC),

675 indirect glutamate pathway (gmaS: $\gamma$-glutamylmethylamide synthase; $\operatorname{mgs} A B C$ : $N$-methyl-L-

676 glutamate synthase; methylglutamate dehydrogenase $\operatorname{mgd} A B C D$ ); dimethylsulfide (DMS)

677 monooxygenase $(d m o A)$, dimethyl sulfone monooxygenase $(s f n G)$, dimethylsulfide

678 monooxygenase (dso), alkane sulfonic acid monooxygenase (ssuD); and dichloromethane

679 dehalogenase $(\mathrm{dcm} A))$. (B) Maximum likelihood phylogenetic tree highlighting the relationship

680 between Binatota methanol dehydrogenases in relation to other methylotrophic taxa. Bootstrap

681 support (from 100 bootstraps) is shown for branches with $>50 \%$. (C) Organization of pMMO

682 genes in Binatota genomes, and the number of genomes where each organization was observed.

683 x: Hypothetical protein (D) Maximum likelihood tree highlighting the relationship between

684 Binatota $p m o A$ genes to methanotrophic taxa and environmental amplicons. Bootstrap support

685 (100 bootstraps) is shown for branches with $>50 \%$ support. Sequences from Binatota genomes

686 (shown as Order followed by Bin name then pmoA protein ID in parentheses) are in magenta and

687 fall into two clusters; Actinobacteria/SAR324 cluster, and TUSC uncultured cluster 2. Clusters

688 from previously studied pMMOs known to reduce methane are in orange, while those known to

689 reduce short chain alkanes but not methane are in cyan (collective data from $28,29,30,131,132,133$ ).

690 The tree was rooted using the amoA sequence of Candidatus Nitrosarchaeum limnium SFB1

691 (EGG41084.1) as an outgroup. (E) Predicted particulate methane monooxygenase (PmoABC)

692 3D structure (grey) from a Cluster 2 TUSC-affiliated Binatota genome (Genome

693 3300027968_51, left), and an Actinobacteria/SAR324-affiliated Binatota genome (Genome 
694 GCA_002238415.1, right) both superimposed on pMMO from the model methanotroph

695 Methylococcus capsulatus str. Bath (pdb: 3RGB) (green) with a global model quality estimate of

6960.7 , and 0.62 , respectively, and a quaternary structure quality score of 0.57 , and 0.55 ,

697 respectively.

698 Figure 3. Formaldehyde oxidation and assimilation capabilities encoded by Binatota genomes.

699 (A) Heatmap of the distribution of formaldehyde oxidation genes in Binatota genomes from

700 different orders. The heatmap colors (as explained in the key) correspond to the percentage of

701 genomes in each order encoding a homologue of the gene in the column header. Shown are the

702 different routes of formaldehyde oxidation, including the (myco)thiol-dependent formaldehyde

703 dehydrogenase $\mathrm{fadH} / \mathrm{mscR}$ (along with mycothiol biosynthesis genes $(m s h A B C)$ ), the $\mathrm{H}_{4} \mathrm{~F}-$ linked

704 pathway (comprising the genes bifunctional methylene- $\mathrm{H}_{4} \mathrm{~F}$ dehydrogenase and methenyl- $\mathrm{H}_{4} \mathrm{~F}$

705 cyclohydrolase $(f o l D)$, reversible formyl- $\mathrm{H}_{4} \mathrm{~F}$ ligase $(f t f L)$, irreversible formyl- $\mathrm{H}_{4} \mathrm{~F}$ hydrolase

$706(\operatorname{pur} U))$, the glutathione-independent formaldehyde dehydrogenase $(f d h A)$, and the glutathione-

707 dependent formaldehyde (comprising the S-(hydroxymethyl)glutathione synthase ( $g f a)$, NAD-

708 and glutathione-dependent formaldehyde dehydrogenase ( $\mathrm{frm} A)$, S-formylglutathione hydrolase

$709(\mathrm{frmB}))$. Also shown is the distribution of the NAD-dependent formate dehydrogenase (EC:

710 1.17.1.9) $(f d h)$ for formate oxidation. (B) Overview of the pathways for formaldehyde

711 assimilation via the serine cycle (left), and glyoxylate regeneration via the ethylmalonyl-CoA

712 pathway and the glyoxylate shunt (GS) (right). Names of enzymes are shown in red and their

713 distribution in the Binatota genomes from different orders is shown in the heatmap in (C). $g l y A$,

714 glycine hydroxymethyltransferase [EC:2.1.2.1]; sgaA; serine-glyoxylate transaminase

715 [EC:2.6.1.45]; $h p r A$, glycerate dehydrogenase [EC:1.1.1.29]; gck, glycerate 2-kinase

716 [EC:2.7.1.165]; $p p c$, phosphoenolpyruvate carboxylase [EC:4.1.1.31]; pckA, 
717 phosphoenolpyruvate carboxykinase; $m d h$, malate dehydrogenase [EC:1.1.1.37]; $m t k A / B$, malate-

718 CoA ligase [EC:6.2.1.9]; $\mathrm{mcl}$, malyl-CoA/(S)-citramalyl-CoA lyase [EC:4.1.3.24 4.1.3.25];

$719 a c e A$, isocitrate lyase [EC:4.1.3.1]; $a c e B$, malate synthase [EC:2.3.3.9]; phbB, acetoacetyl-CoA

720 reductase [EC:1.1.1.36]; croR, 3-hydroxybutyryl-CoA dehydratase [EC:4.2.1.55]; ccr, crotonyl-

721 CoA carboxylase/reductase [EC:1.3.1.85]; epi, methylmalonyl-CoA/ethylmalonyl-CoA

722 epimerase [EC:5.1.99.1]; ecm, ethylmalonyl-CoA mutase [EC:5.4.99.63]; $\mathrm{mcd},(2 \mathrm{~S})-$

723 methylsuccinyl-CoA dehydrogenase [EC:1.3.8.12]; mch, 2-methylfumaryl-CoA hydratase

724 [EC:4.2.1.148]; mut, methylmalonyl-CoA mutase [EC:5.4.99.2]; mcmA1/A2, methylmalonyl-

725 CoA mutase [EC:5.4.99.2]. Abbreviations: PEP, phosphoenol pyruvate; OAA, oxaloacetate.

726 Figure 4. Alkane, and fatty acid degradation capabilities encoded in Binatota genomes. (A)

727 Heatmap of the distribution of (halo)alkane degradation to alcohol. The heatmap colors (as

728 explained in the key) correspond to the percentage of genomes in each order encoding a

729 homologue of the gene in the column header. The per-order predicted alkane degradation

730 capacity is shown to the right as a heatmap with the colors corresponding to the percentage of

731 genomes in each order where the full degradation pathway was detected for the substrate in the

732 column header. These include $p m o A B C$ and/or $p r m A B C$ for short-chain alkanes, alkB, or cyp153

733 for medium-chain alkanes, ladA for long-chain alkanes, and dhaA for haloalkanes. (B) Heatmap

734 of the distribution of various chain-length fatty acid and haloacid degradation genes in Binatota

735 genomes. The heatmap colors (as explained in the key) correspond to the percentage of genomes

736 in each order encoding a homologue of the gene in the column header. (C) Propionyl-CoA

737 degradation pathways encoded by the Binatota genomes. The methylmalonyl CoA (MMCoA)

738 pathway is shown in blue, while the 2-methylcitrate pathway is shown in green. In some

739 genomes, the MMCoA pathway seems to be functional but with a slight modification (shown in 
purple) that includes glyoxylate assimilation and regeneration. pmo $A B C$, particulate methane

741 monooxygenase with denoting subunits $\mathrm{A}, \mathrm{B}$, and $\mathrm{C}$; prm $A B C$, propane 2-monooxygenase

742 [EC:1.14.13.227]; alkB, alkane 1-monooxygenase [EC:1.14.15.3]; cyp153, Cytochrome P450

743 alkane hydroxylase [EC 1.14.15.1]; ladA, long-chain alkane monooxygenase [EC:1.14.14.28];

$744 d h a A$, haloalkane dehalogenase [EC:3.8.1.5]; adh, alcohol dehydrogenase [EC:1.1.1.1];

745 EC:1.1.1.80, isopropanol dehydrogenase (NADP+) [EC:1.1.1.80]; acmA, acetone

746 monooxygenase (methyl acetate-forming) [EC:1.14.13.226]; acmB, methyl acetate hydrolase

747 [EC:3.1.1.114]; EC:1.2.1.3, aldehyde dehydrogenase (NAD+) [EC:1.2.1.3]; E1.2.1.10, 748 acetaldehyde dehydrogenase (acetylating) [EC:1.2.1.10]; acdAB, acetate---CoA ligase (ADP-

749 forming) [EC:6.2.1.13]; acs, acetyl-CoA synthase [EC:2.3.1.169]; atoAD, acetate

$750 \mathrm{CoA} /$ acetoacetate CoA-transferase [EC:2.8.3.8 2.8.3.9]; EC:6.2.1.2, medium-chain acyl-CoA

751 synthetase [EC:6.2.1.2]; fadD, long-chain acyl-CoA synthetase [EC:6.2.1.3]; pccA, propionyl-

752 CoA carboxylase alpha chain [EC:6.4.1.3]; epi, methylmalonyl-CoA/ethylmalonyl-CoA

753 epimerase [EC:5.1.99.1]; mut, methylmalonyl-CoA mutase [EC:5.4.99.2]; mcl, malyl-CoA/(S)-

754 citramalyl-CoA lyase [EC:4.1.3.24 4.1.3.25]; mch, 2-methylfumaryl-CoA hydratase

755 [EC:4.2.1.148]; mct, 2-methylfumaryl-CoA isomerase [EC:5.4.1.3]; meh, 3-methylfumaryl-CoA

756 hydratase [EC:4.2.1.153]; smtAB, succinyl-CoA:(S)-malate CoA-transferase subunit A

757 [EC:2.8.3.22]; $\operatorname{prpB}$, methylisocitrate lyase [EC:4.1.3.30]; prpC, 2-methylcitrate synthase

758 [EC:2.3.3.5]; prpD, 2-methylcitrate dehydratase [EC:4.2.1.79]; bcd, butyryl-CoA dehydrogenase

759 [EC:1.3.8.1]; acd, acyl-CoA dehydrogenase [EC:1.3.8.7]; paaF, enoyl-CoA hydratase

760 [EC:4.2.1.17]; crt, enoyl-CoA hydratase [EC:4.2.1.17]; paaH, 3-hydroxybutyryl-CoA

761 dehydrogenase [EC:1.1.1.157]; phbB, acetoacetyl-CoA reductase [EC:1.1.1.36]; atoB, acetyl-

762 CoA C-acetyltransferase [EC:2.3.1.9]; fadJ, 3-hydroxyacyl-CoA dehydrogenase / enoyl-CoA 
763 hydratase / 3-hydroxybutyryl-CoA epimerase [EC:1.1.1.35 4.2.1.17 5.1.2.3]; fadA, acetyl-CoA

764 acyltransferase [EC:2.3.1.16]; dehH, 2-haloacid dehalogenase [EC:3.8.1.2]; EC:3.8.1.3,

765 haloacetate dehalogenase [EC:3.8.1.3]; glcDEF, glycolate oxidase [EC:1.1.3.15]; EC:1.1.3.15,

766 (S)-2-hydroxy-acid oxidase [EC:1.1.3.15].

767 Figure 5. Electron transport chain in the Binatota. (A) Heatmap of the distribution of electron

768 transport chain components in the Binatota genomes and electrons entry points from various

769 substrates. The heatmap colors (as explained in the key) correspond to the percentage of

770 genomes in each order encoding a homologue of the gene in the column header. All subunits of

771 complexes I (NADH-quinone oxidoreductase [EC:7.1.1.2]), and II (succinate dehydrogenase

772 /fumarate reductase [EC:1.3.5.1 1.3.5.4]) were encoded in all genomes but are shown here as

773 single components for ease of visualization. Genes encoding quinone-cytochrome C reductase

774 activities belonged to either complex III (cytochrome bc1; ISP/cytb/cyt1) and/or alternate

775 cytochrome III (ACIII; actABCDEF), while genes encoding cytochrome c oxidase activities

776 (complex IV) belonged to different families including family A (cytochrome c oxidase aa3;

$777 \operatorname{cox} A B C$ ), family $\mathrm{C}$ (cytochrome c oxidase cbb3; ccoNOP), and/or cytochrome $b d(c y d A B)$.

778 Possible electron transfer proteins between complex III (or alternate complex III) and complex

779 IV belonging to different cytochrome c families are shown. Also shown in (A) is the distribution

780 of the three subunits of the type I respiratory $\mathrm{O}_{2}$-tolerant $\mathrm{H}_{2}$-uptake $[\mathrm{NiFe}$ hydrogenase

781 (hyaABC) in Binatota genomes. (B) A cartoon depicting all electron transfer complexes (I, II,

782 ACIII, IV) embedded in the inner membrane, along with the particulate methane monooxygenase

783 (pMMO), and the $\mathrm{H}_{2}$-uptake $[\mathrm{NiFe}]$ hydrogenase (HyaABC). All genomes also encoded an F-

784 type ATP synthase complex (V). Substrates potentially supporting growth are shown in blue with

785 predicted entry points to the ETC shown as dotted black arrows. Sites of proton extrusion to the 
786 periplasm and PMF creation are shown as solid black lines, while sites of electron (e') transfer

787 are shown as dotted green lines. Three possible physiological reductants are shown for pMMO

788 (as dotted green arrows); the quinone pool coupled to ACIII, NADH, and/or some of the reduced

789 quinones generated through $\mathrm{H}_{2}$ oxidation by HyaABC. (C) Maximum likelihood phylogenetic

790 tree showing the classification of the hyaA genes encoded by the Binatota genomes (magenta) in

791 relation to other [Ni-Fe] hydrogenases. The [Fe-Fe] hydrogenase of Methanobacterium formicum

792 was used as the outgroup. Bootstrap support (from 100 bootstraps) is shown for branches

793 with $>50 \%$ support.

794 Figure 6. Carotenoids biosynthesis capabilities in Binatota genomes. (A) Distribution of

795 carotenoid biosynthesis genes in the Binatota genomes. The heatmap colors (as explained in the

796 key) correspond to the percentage of genomes in each order encoding a homologue of the gene in

797 the column header. (B) Carotenoid biosynthesis scheme in Binatota based on the identified

798 genes. Genes encoding enzymes catalyzing each step are shown in red and their descriptions

799 with EC numbers are shown to the right. Binatota genomes encode the capability to

800 biosynthesize both exclusively hydrocarbon carotenes (white boxes), or the oxygenated

801 xanthophylls (grey boxes).

802 Figure 7. Bacteriochlorophylls biosynthesis genes encountered in Binatota genomes studied

803 suggesting an incomplete pathway for bacteriochlorophyll $a, c$, and/or $d$ biosynthesis.

804 (A) Distribution of chlorophyll biosynthesis genes in Binatota genomes. The heatmap colors (as

805 explained in the key) correspond to the percentage of genomes in each order encoding a

806 homologue of the gene in the column header. (B) Bacteriochlorophylls biosynthesis pathway.

807 Genes identified in at least one Binatota genome are shown in red boldface text, while these with

808 no homologues in the Binatota genomes are shown in blue text. Gene descriptions with EC 
numbers are shown to the right of the figure. (C) Distribution patterns of bacteriochlorophyll

810 biosynthesis genes. The search was conducted in the functionally annotated bacterial tree of life

811 AnnoTree ${ }^{96}$ using single KEGG orthologies implicated in chlorophyll biosynthesis. Gene names

812 are shown on the $\mathrm{X}$-axis, total number of hits are shown above the bars for each gene, and the

813 percentage of hits in genomes from photosynthetic $(\square)$ versus non-photosynthetic $(\square)$ genera

814 are in the stacked bars.

815 Figure 8. Ecological distribution of Binatota-affiliated 16S rRNA sequences in GenBank nt

816 database. Binatota orders are shown on the X-axis, while percentage abundance in different

817 environments (classified based on the GOLD ecosystem classification scheme) are shown on the

818 Y-axis (A). Further sub-classifications for each environment are shown for (B) terrestrial, (C)

819 freshwater, (D) marine, (E) host-associated, and (F) engineered environments. The total number

820 of hit sequences for each order are shown above the bar graphs. Details including GenBank

821 accession number of hit sequences are shown in Extended Data 2. Order UBA12015 genome

822 assembly did not contain a16S rRNA gene, and so this order is not included in the analysis.

823 Figure 9. Cartoon depicting different metabolic capabilities encoded in the Binatota genomes.

824 Enzymes for $\mathrm{C} 1$ metabolism are shown in blue and include the periplasmic particulate methane

825 monooxygenase (pMMO), methanol dehydrogenase ( $x \circ F F$ ), and methylamine dehydrogenase

826 (mauABC), as well as the cytoplasmic formaldehyde dehydrogenase (FalDH), and formate

827 dehydrogenase (FDH). Electron transport chain is shown as a green rectangle. Electron transfer

828 from periplasmic enzymes to the ETC is shown as dotted green lines (details of the ETC are

829 shown in Figure 5b). The sites of proton extrusion to the periplasm are shown as black arrows, as

830 is the F-type ATP synthase. Carbon dissimilation routes are shown as red arrows, while

831 assimilatory routes are shown as purple arrows. Details of the assimilatory pathways are shown 
832 in Figures 2 and 3. Reducing equivalents potentially fueling the ETC $(\mathrm{NAD}(\mathrm{P}) \mathrm{H}$, and $\mathrm{FADH} 2)$

833 are shown in boldface. All substrates predicted to support growth are shown in boldface within

834 grey boxes. A flagellum is also depicted, the biosynthetic genes of which were identified in

835 genomes belonging to all orders except Bin18, HRBin30, and UBA1149. The cell is also

836 depicted as rod-shaped based on the identification of the rod shape determining protein $\operatorname{rod} A$ in

837 all genomes, and the rod-shape determining proteins $m r e B$ and $m r e C$ in genomes from all orders

838 except UBA1149. Abbreviations: CBB, Calvin Bensen Basham cycle; Fal-DH, NAD-linked

839 glutathione-independent formaldehyde dehydrogenase, $f d h A$; FDH, NAD-dependent formate

840 dehydrogenase [EC: 1.17.1.9); Fum, fumarate; GS, glyoxylate shunt; $\mathrm{H}_{4} \mathrm{~F}$, tetrahydrofolate;

841 HyaABC, type I respiratory $\mathrm{O}_{2}$-tolerant $\mathrm{H}_{2}$-uptake $[\mathrm{NiFe}]$ hydrogenase; mauABC, methylamine

842 dehydrogenase; $p m o A B C$, particulate methane monooxygenase; $x o x F G$, xoxF-type methanol

843 dehydrogenase; succ, succinate; TCA, tricarboxylic acid cycle; V, F-type ATP synthase

$844 \quad[\mathrm{EC}: 7.1 .2 .2$ 7.2.2.1]. 


\section{References}

846 1. Hug LA, et al. Critical biogeochemical functions in the subsurface are associated with

2. Engelberts JP, Robbins SJ, de Goeij JM, Aranda M, Bell SC, Webster NS.

3. Vavourakis CD, et al. Metagenomes and metatranscriptomes shed new light on the microbial-mediated sulfur cycle in a Siberian soda lake. BMC Biol. 17, 69 (2019).

4. $\mathrm{Hu} \mathrm{P}$, et al. Simulation of Deepwater Horizon oil plume reveals substrate specialization within a complex community of hydrocarbon degraders. Proc. Natl. Acad. Sci. USA 114, $7432-7437$ (2017).

5. Doud DFR, et al. Function-driven single-cell genomics uncovers cellulose-degrading bacteria from the rare biosphere. ISME J14, 659-675 (2019).

6. Anantharaman $\mathrm{K}$, et al. Expanded diversity of microbial groups that shape the dissimilatory sulfur cycle. ISME J 12, 1715-1728 (2018).

7. Becraft ED, et al. Rokubacteria: Genomic giants among theuncultured bacterial phyla. Front. Micorobiol. 8, 2264 (2017).

8. Rinke R, et al. A phylogenomic and ecological analysis of the globally abundant Marine Group II archaea (Ca. Poseidoniales ord. nov.). ISME J. 13, 663-675 (2019).

9. Farag IF, Davis JP, Youssef NH, Elshahed MS. Global patterns of abundance, diversity and community structure of the Aminicenantes (Candidate Phylum OP8). PloS one 9, e92139 (2014).

10. Zhou Z, Tran PQ, Kieft K, Anantharaman K. Genome diversification in globally distributed novel marine Proteobacteria is linked to environmental adaptation. ISME J. 14, 2060-2077 (2020).

11. Youssef NH, Blainey PC, Quake SR, Elshahed MS. Partial genome assembly for a candidate division OP11 single cell from an anoxic spring (Zodletone Spring, Oklahoma). Appl. Environ. Microbiol. 77, 7804-7814 (2011).

12. Rinke $\mathrm{C}$, et al. Insights into the phylogeny and coding potential of microbial dark matter. Nature 499, 431-437 (2013). 
14. Parks DH, et al. A standardized bacterial taxonomy based on genome phylogeny substantially revises the tree of life. Nat. Biotechnol. 36, 996-1004 (2018)

15. Parks DH, et al. Recovery of nearly 8,000 metagenome-assembled genomes substantially

16. Nayfach S, et al. A genomic catalogue of Earth's microbiomes. Nat. Biotechnol. Accpeted, (2020).

17. Chuvochina M, et al. The importance of designating type material for uncultured taxa.

18. Bowers RM, et al. Minimum information about a single amplified genome (MISAG) and a metagenome-assembled genome (MIMAG) of bacteria and archaea. Nat. Biotechnol. 35, 725-731 (2017).

19. Quast C, et al. The SILVA ribosomal RNA gene database project: improved data

20. Hektor HJ, Kloosterman H, Dijkhuizen L. Nicotinoprotein methanol dehydrogenase

21. Kalyuzhnaya MG, Hristova KR, Lidstrom ME, Chistoserdova L. Characterization of a novel methanol dehydrogenase in representatives of Burkholderiales: implications for environmental detection of methylotrophy and evidence for convergent evolution. $J$. Bacteriol. 190, 3817-3823 (2008).

22. Chistoserdova L. Modularity of methylotrophy, revisited. Environ. Microbiol. 13, 26032622 (2011).

23. Erikstad HA, Jensen S, Keen TJ, Birkeland NK. Differential expression of particulate methane monooxygenase genes in the verrucomicrobial methanotroph 'Methylacidiphilum kamchatkense' Kam1. Extremophiles 16, 405-409 (2012).

24. Ettwig KF, et al. Nitrite-driven anaerobic methane oxidation by oxygenic bacteria. Nature 464, 543-548 (2010).

25. Iguchi H, Yurimoto H, Sakai Y. Soluble and particulate methane monooxygenase gene clusters of the type I methanotroph Methylovulum miyakonense HT12. FEMS Microbiol. Methylocystis strain SC2. Appl. Environ. Microbiol. 70, 3055-3063 (2004). 
27. Fisher OS, et al. Characterization of a long overlooked copper protein from methane- and ammonia-oxidizing bacteria. Nat. Commun. 9, 4276 (2018).

28. Rochman FF, et al. Novel copper-containing membrane monooxygenases (CuMMOs) encoded by alkane-utilizing Betaproteobacteria. ISME J. 14, 714-726 (2020).

29. Knief C. Diversity and habitat preferences of cultivated and uncultivated aerobic methanotrophic bacteria evaluated based on pmoA as molecular marker. Front. Microbiol. 6, 1346 (2015).

30. Li M, Jain S, Baker BJ, Taylor C, Dick GJ. Novel hydrocarbon monooxygenase genes in the metatranscriptome of a natural deep-sea hydrocarbon plume. Environ. Microbiol. 16, 60-71 (2014).

31. Sheik CS, Jain S, Dick GJ. Metabolic flexibility of enigmatic SAR324 revealed through metagenomics and metatranscriptomics. Environ. Microbiol. 16, 304-317 (2014).

32. Kalyuzhnaya MG, Zabinsky R, Bowerman S, Baker DR, Lidstrom ME, Chistoserdova L. Fluorescence in situ hybridization-flow cytometry-cell sorting-based method for separation and enrichment of type I and type II methanotroph populations. Appl. Environ. Microbiol. 72, 4293-4301 (2006).

33. Hamamura N, Yeager CM, Arp DJ. Two distinct monooxygenases for alkane oxidation in Nocardioides sp. strain CF8. Appl. Environ. Microbiol. 67, 4992-4998 (2001).

34. Lessmeier L, Hoefener M, Wendisch VF. Formaldehyde degradation in Corynebacterium glutamicum involves acetaldehyde dehydrogenase and mycothiol-dependent formaldehyde dehydrogenase. Microbiology (Reading, England) 159, 2651-2662 (2013).

35. Dubey AA, Wani SR, Jain V. Methylotrophy in Mycobacteria: dissection of the methanol metabolism pathway in Mycobacterium smegmatis. J. Bacteriol. 200, e00288-18 (2018).

36. Kornberg HL, Krebs HA. Synthesis of cell constituents from C2-units by a modified tricarboxylic acid cycle. Nature 179, 988-991 (1957).

37. Alber BE, Spanheimer R, Ebenau-Jehle C, Fuchs G. Study of an alternate glyoxylate cycle for acetate assimilation by Rhodobacter sphaeroides. Mol. Microbiol. 61, 297-309 (2006).

38. Chen Q, Janssen DB, Witholt B. Growth on octane alters the membrane lipid fatty acids of Pseudomonas oleovorans due to the induction of alkB and synthesis of octanol. $J$. Bacteriol. 177, 6894-6901 (1995).

39. van Beilen JB, Funhoff EG. Alkane hydroxylases involved in microbial alkane degradation. Appl. Microbiol. Biotechnol. 74, 13-21 (2007). 
1002

1003

1004

1005

1006

1007

1008

1009

1010

1011

1012

1013

1014

1015

1016

1017

1018

1019

1020

1021

1022

1023

1024

1025

1026

1027

1028

40. Li L, et al. Crystal structure of long-chain alkane monooxygenase (LadA) in complex with coenzyme FMN: unveiling the long-chain alkane hydroxylase. J. Mol. Biol. 376, 453-465 (2008).

41. Nagata Y, Miyauchi K, Damborsky J, Manova K, Ansorgova A, Takagi M. Purification and characterization of a haloalkane dehalogenase of a new substrate class from a gamma-hexachlorocyclohexane-degrading bacterium, Sphingomonas paucimobilis UT26. Appl. Environ. Microbiol. 63, 3707-3710 (1997).

42. Sun C, et al. Structure of the alternative complex III in a supercomplex with cytochrome oxidase. Nature 557, 123-126 (2018).

43. Choi DW, et al. The membrane-associated methane monooxygenase (pMMO) and pMMO-NADH:quinone oxidoreductase complex from Methylococcus capsulatus Bath. J. Bacteriol.y 185, 5755-5764 (2003).

44. Nguyen HH, Elliott SJ, Yip JH, Chan SI. The particulate methane monooxygenase from Methylococcus capsulatus (Bath) is a novel copper-containing three-subunit enzyme. Isolation and characterization. J. Biol. Chem. 273, 7957-7966 (1998).

45. Wulff P, Day CC, Sargent F, Armstrong FA. How oxygen reacts with oxygen-tolerant USA 111, 6606-6611 (2014).

46. Sargent F. The Model [NiFe]-Hydrogenases of Escherichia coli. Adv. Microb. Physiol. 68, 433-507 (2016).

47. Volbeda A, Darnault C, Parkin A, Sargent F, Armstrong FA, Fontecilla-Camps JC. Crystal structure of the $\mathrm{O}(2)$-tolerant membrane-bound hydrogenase 1 from Escherichia coli in complex with its cognate cytochrome b. Structure 21, 184-190 (2013).

48. Carere CR, et al. Mixotrophy drives niche expansion of verrucomicrobial methanotrophs. ISME J. 11, 2599-2610 (2017).

49. Chistoserdova L, Kalyuzhnaya MG, Lidstrom ME. The expanding world of methylotrophic metabolism. Ann. Rev. Microbiol. 63, 477-499 (2009).

50. Boden R, Thomas E, Savani P, Kelly DP, Wood AP. Novel methylotrophic bacteria isolated from the River Thames (London, UK). Environ. Microbiol. 10, 3225-3236 (2008).

51. McTaggart TL, et al. Genomics of methylotrophy in Gram-positive methylamineutilizing bacteria. Microorganisms 3, 94-112 (2015).

52. Pol A, Heijmans K, Harhangi HR, Tedesco D, Jetten MSM, Op den Camp HJM. Methanotrophy below pH 1 by a new Verrucomicrobia species. Nature 450, 874-878 (2007). 
53. Ettwig KF, van Alen T, van de Pas-Schoonen KT, Jetten MS, Strous M. Enrichment and molecular detection of denitrifying methanotrophic bacteria of the NC10 phylum. Appl. Environ. Microbiol. 75, 3656-3662 (2009).

54. Diamond S, et al. Mediterranean grassland soil C-N compound turnover is dependent on rainfall and depth, and is mediated by genomically divergent microorganisms. Nat. Microbiol. 4, 1356-1367 (2019).

55. Butterfield CN, et al. Proteogenomic analyses indicate bacterial methylotrophy and archaeal heterotrophy are prevalent below the grass root zone. PeerJ 4, e2687 (2016).

56. Davamani V, Parameswari E, Arulmani S. Mitigation of methane gas emissions in flooded paddy soil through the utilization of methanotrophs. Sci Tot. Environ. 726, 138570 (2020).

57. Khmelenina VN, Colin Murrell J, Smith TJ, Trotsenko YA. Physiology and biochemistry of the aerobic methanotrophs. In: Aerobic utilization of hydrocarbons, oils and lipids (ed Rojo F). Springer International Publishing (2018).

58. Mohammadi SS, Pol A, van Alen T, Jetten MSM, Op den Camp HJM. Ammonia oxidation and nitrite reduction in the verrucomicrobial methanotroph Methylacidiphilum fumariolicum SolV. Front. Microbiol. 8, 1901 (2017).

59. Prince RC, Amande TJ, McGenity TJ. Prokaryotic hydrocarbon degraders. In: Taxonomy, genomics and ecophysiology of hydrocarbon-degrading microbes (ed McGenity TJ). Springer International Publishing (2019).

60. Le Mer J, Roger P. Production, oxidation, emission and consumption of methane by soils: A review. Eur. J. Soil Biol. 37, 25-50 (2001).

61. Wang Z, Zeng D, Patrick WH. Methane emissions from natural wetlands. Environ. Monit. Assess.42, 143-161 (1996).

62. He S, et al. Patterns in wetland microbial community composition and functional gene repertoire associated with methane emissions. mBio 6, e00066-00015 (2015).

63. Angle JC, et al. Methanogenesis in oxygenated soils is a substantial fraction of wetland methane emissions. Nat. Commun. 8, 1567 (2017).

64. Kolb S. Aerobic methanol-oxidizing Bacteria in soil. FEMS Microbiol. Lett. 300, 1-10 (2009).

65. Conrad R. The global methane cycle: recent advances in understanding the microbial processes involved. Environ. Microbiol. Rep. 1, 285-292 (2009). 
1075

1076

1077

1078

1079

1080

1081

1082

1083

1084

1085

1086

1087

1088

1089

1090

1091

1092

1093

1094

1095

1096

1097

1098

1099

1100

1101

1102

1103

1104

1105

1106

1107

1108

1109

1110

1111

1112

1113

1114

1115

1116

1117

1118

1119

1120

66. McCollom TM. Laboratory simulations of abiotic hydrocarbon formation in earth's deep subsurface. Rev.n Mineral. Geochem. 75, 467-494 (2013).

67. Wang W, Li Z, Zeng L, Dong C, Shao Z. The oxidation of hydrocarbons by diverse heterotrophic and mixotrophic bacteria that inhabit deep-sea hydrothermal ecosystems. ISME J. 14, 1994-2006 (2020).

68. Pasche N, et al. Methane sources and sinks in Lake Kivu. J. Geophys. Res. Biogeosci. 116, G03006 (2011).

69. Borges AV, Abril G, Delille B, Descy J-P, Darchambeau F. Diffusive methane emissions to the atmosphere from Lake Kivu (Eastern Africa). J. Geophys. Res. Biogeosci. 116, G03032 (2011).

70. Llirós M, et al. Microbial Ecology of Lake Kivu. In: Lake Kivu: Limnology and biogeochemistry of a tropical great lake (eds Descy J-P, Darchambeau F, Schmid M). Springer Netherlands (2012).

71. Chistoserdova L. Methylotrophy in a lake: from metagenomics to single-organism physiology. Appl. Environ. Microbiol. 77, $4705-4711$ (2011).

72. Chistoserdova L. The distribution and evolution of $\mathrm{c} 1$ transfer enzymes and evolution of the Planctomycetes. In: Planctomycetes: Cell Structure, Origins and Biology (ed Fuerst JA). Humana Press (2013).

73. Auman AJ, Lidstrom ME. Analysis of sMMO-containing type I methanotrophs in Lake Washington sediment. Environ. Microbiol. 4, 517-524 (2002).

74. Auman AJ, Stolyar S, Costello AM, Lidstrom ME. Molecular characterization of methanotrophic isolates from freshwater lake sediment. Appl. Environ. Microbiol. 66, 5259-5266 (2000).

75. Chistoserdova L. Methylotrophs in natural habitats: current insights through metagenomics. Appl. Microbiol. Biotechnol. 99, 5763-5779 (2015).

76. Kuivila KM, Murray JW, Devol AH, Lidstrom ME, Reimers CE. Methane cycling in the sediments of Lake Washington. Limnol. Oceanogr. 33, 571-581 (1988).

77. Arellano SM, et al. Deep sequencing of Myxilla (Ectyomyxilla) methanophila, an epibiotic sponge on cold-seep tubeworms, reveals methylotrophic, thiotrophic, and putative hydrocarbon-degrading microbial associations. Microb. Ecol. 65, 450-461 (2013).

78. Tian RM, Zhang W, Cai L, Wong YH, Ding W, Qian PY. Genome reduction and microbe-host interactions drive adaptation of a sulfur-oxidizing bacterium associated with a cold seep sponge. mSystems 2, (2017). 
79. Rubin-Blum M, et al. Fueled by methane: deep-sea sponges from asphalt seeps gain their nutrition from methane-oxidizing symbionts. ISME J. 13, 1209-1225 (2019).

80. Hashimoto H, Uragami C, Cogdell RJ. Carotenoids and photosynthesis. Subcell. Biochem. 79, 111-139 (2016).

81. Saidi-Mehrabad A, et al. Methylicorpusculum oleiharenae gen. nov., sp. nov., an aerobic methanotroph isolated from an oil sands tailings pond. Int. J. Syst. Evol. Microbiol. 70, 2499-2508 (2020).

82. Wang FQ, et al. Carboxylicivirga sediminis sp. nov., isolated from coastal sediment. Int. J. Syst. Evol. Microbiol. 68, 1896-1901 (2018).

83. Asker D, Awad TS, Beppu T, Ueda K. Deinococcus misasensis and Deinococcus roseus, novel members of the genus Deinococcus, isolated from a radioactive site in Japan. Syst. Appl. Microbiol. 31, 43-49 (2008).

84. Zhou EM, et al. Thermus sediminis sp. nov., a thiosulfate-oxidizing and arsenatereducing organism isolated from Little Hot Creek in the Long Valley Caldera, California. Extremophiles 22, 983-991 (2018).

85. Sanford RA, Cole JR, Tiedje JM. Characterization and description of Anaeromyxobacter dehalogenans gen. nov., sp. nov., an aryl-halorespiring facultative anaerobic myxobacterium. Appl. Environ. Microbiol. 68, 893-900 (2002).

86. Fariq A, Yasmin A, Jamil M. Production, characterization and antimicrobial activities of bio-pigments by Aquisalibacillus elongatus MB592, Salinicoccus sesuvii MB597, and Halomonas aquamarina MB598 isolated from Khewra Salt Range, Pakistan. Extremophiles 23, 435-449 (2019).

87. Ungers GE, Cooney JJ. Isolation and characterization of carotenoid pigments of Micrococcus roseus. J. Bacteriol. 96, 234-241 (1968).

88. Bondoso J, Albuquerque L, Nobre MF, Lobo-da-Cunha A, da Costa MS, Lage OM. Roseimaritima ulvae gen. nov., sp. nov. and Rubripirellula obstinata gen. nov., sp. nov. two novel planctomycetes isolated from the epiphytic community of macroalgae. Syst. Appl. Microbiol.38, 8-15 (2015).

89. Chen S, Sun S, Xu Y, Liu HC. Halococcus salsus sp. nov., a novel halophilic archaeon isolated from rock salt. Int. J. Syst. Evol. Microbiol. 68, 3754-3759 (2018).

90. Grogan DW. Phenotypic characterization of the archaebacterial genus Sulfolobus: comparison of five wild-type strains. J. Bacteriol. 171, 6710-6719 (1989). 
91. Fiedor J, Sulikowska A, Orzechowska A, Fiedor L, Burda K. Antioxidant effects of carotenoids in a model pigment-protein complex. Acta Biochim. Polon. 59, 61-64 (2012).

92. Krisko A, Radman M. Biology of extreme radiation resistance: the way of Deinococcus radiodurans. Cold Spring Harbor Pers. Biol. 5, a012765 (2013).

93. Du X-j, Wang X-y, Dong X, Li P, Wang S. Characterization of the desiccation tolerance of Cronobacter sakazakii strains. Front Microbiol. 9, 2867 (2018).

94. Bowman JP, Sly LI, Nichols PD, Hayward AC. Revised taxonomy of the methanotrophs: Description of Methylobacter gen. nov., Emendation of Methylococcus, Validation of Methylosinus and Methylocystis Species, and a proposal that the family Methylococcaceae includes only the group I methanotrophs. Int. J. Syst. Evol. Microbiol.

95. Irvine IC, Brigham CA, Suding KN, Martiny JB. The abundance of pink-pigmented facultative methylotrophs in the root zone of plant species in invaded coastal sage scrub habitat. PloS one 7, e31026 (2012).

1184

96. Mendler K, Chen H, Parks DH, Lobb B, Hug LA, Doxey AC. AnnoTree: visualization and exploration of a functionally annotated microbial tree of life. Nucl. Acids Res. 47, 4442-4448 (2019).

97. Meng J, et al. An uncultivated crenarchaeota contains functional bacteriochlorophyll a synthase. ISME J. 3, 106-116 (2009).

98. Liu R, Cai R, Zhang J, Sun C. Heimdallarchaeota harness light energy through photosynthesis. bioRxiv, 2020.2002.2020.957134 (2020).

1194

99. Martin WF, Bryant DA, Beatty JT. A physiological perspective on the origin and evolution of photosynthesis. FEMS Mmicrobiol. Rev. 42, 205-231 (2018).

100. White SN, Chave AD, Reynolds GT, Van Dover CL. Ambient light emission from hydrothermal vents on the Mid-Atlantic Ridge. Geophys. Res. Lett. 29, 34-31-34-34 (2002).

101. Gao X, Xin Y, Bell PD, Wen J, Blankenship RE. Structural analysis of alternative complex III in the photosynthetic electron transfer chain of Chloroflexus aurantiacus. Biochemistry 49, 6670-6679 (2010).

102. Chistoserdova L, Lidstrom ME. Aerobic methylotrophic prokaryotes. In: The Prokaryotes: prokaryotic physiology and biochemistry (eds Rosenberg E, DeLong EF, Lory S, Stackebrandt E, Thompson F). Springer Berlin Heidelberg (2013).

103. Quayle JR, Pfennig N. Utilization of methanol by rhodospirillaceae. Arch. Microbiol. 102, 193-198 (1975). 
104. Anantharaman K, et al. Thousands of microbial genomes shed light on interconnected biogeochemical processes in an aquifer system. Nat. Commun. 7, 13219 (2016).

105. Hug LA, Co R. It takes a village: microbial communities thrive through interactions and metabolic handoffs. mSystems 3, e00152-00117 (2018).

106. Colman DR, Lindsay MR, Amenabar MJ, Fernandes-Martins MC, Roden ER, Boyd ES. Phylogenomic analysis of novel Diaforarchaea is consistent with sulfite but not sulfate reduction in volcanic environments on early Earth. ISME J. 14, 1316-1331 (2020).

107. Garcia SL, Buck M, McMahon KD, Grossart H-P, Eiler A, Warnecke F. Auxotrophy and intrapopulation complementary in the 'interactome' of a cultivated freshwater model community. Mol. Ecol. 24, 4449-4459 (2015).

108. Croft MT, Lawrence AD, Raux-Deery E, Warren MJ, Smith AG. Algae acquire vitamin B12 through a symbiotic relationship with bacteria. Nature 438, 90-93 (2005).

109. Parks DH, Imelfort M, Skennerton CT, Hugenholtz P, Tyson GW. CheckM: assessing the quality of microbial genomes recovered from isolates, single cells, and metagenomes. Genome Res. 25, 1043-1055 (2015).

110. Parks DH, Chuvochina M, Chaumeil PA, Rinke C, Mussig AJ, Hugenholtz P. A complete domain-to-species taxonomy for Bacteria and Archaea. Nat. Biotechnol. 38,1079-1086 (2020).

111. Chaumeil PA, Mussig AJ, Hugenholtz P, Parks DH. GTDB-Tk: a toolkit to classify genomes with the Genome Taxonomy Database. Bioinformatics 36, 1925-1927 (2019).

112. Stamatakis A. RAxML version 8: a tool for phylogenetic analysis and post-analysis of large phylogenies. Bioinformatics 30, 1312-1313 (2014).

113. Lagesen K, Hallin P, Rødland EA, Staerfeldt HH, Rognes T, Ussery DW. RNAmmer: consistent and rapid annotation of ribosomal RNA genes. Nucl. Acids Res. 35, 3100-3108 (2007).

114. Hyatt D, Chen GL, Locascio PF, Land ML, Larimer FW, Hauser LJ. Prodigal: prokaryotic gene recognition and translation initiation site identification. $B M C$ Bioinformatics 11, 119 (2010).

115. Bayliss SC, Thorpe HA, Coyle NM, Sheppard SK, Feil EJ. PIRATE: A fast and scalable pangenomics toolbox for clustering diverged orthologues in bacteria. GigaScience $\mathbf{8}$, giz119 (2019). 
116. Kanehisa M, Sato Y, Morishima K. BlastKOALA and GhostKOALA: KEGG Tools for Functional Characterization of Genome and Metagenome Sequences. J. Mol. Biol. 428, 726-731 (2016).

117. Kanehisa M, Sato Y. KEGG Mapper for inferring cellular functions from protein sequences. Prot. Sci. 29, 28-35 (2020).

118. Sievers F, Higgins DG. Clustal Omega for making accurate alignments of many protein sequences. Prot. Sci. 27, 135-145 (2018).

119. Price MN, Dehal PS, Arkin AP. FastTree 2--approximately maximum-likelihood trees for large alignments. PloS one 5, e9490 (2010).

120. Orf GS, Gisriel C, Redding KE. Evolution of photosynthetic reaction centers: insights from the structure of the heliobacterial reaction center. Photosyn. Res. 138, 11-37 (2018).

121. Tsuji J, et al. Anoxygenic phototrophic Chloroflexota member uses a Type I reaction center. bioRxiv, 2020.2007.2007.190934 (2020).

122. Sadekar S, Raymond J, Blankenship RE. Conservation of distantly related membrane proteins: photosynthetic reaction centers share a common structural core. Mol. Biol. Evol. 23, 2001-2007 (2006).

123. Waterhouse A, et al. SWISS-MODEL: homology modelling of protein structures and complexes. Nucl. Acids Res. 46, W296-w303 (2018).

124. Hohmann-Marriott MF, Blankenship RE. Evolution of photosynthesis. Ann. Rev. Plant Biol. 62, 515-548 (2011).

125. Krauß N. Structure and function of cyanobacterial photosystem I. In: Photosynthetic Protein Complexes (ed Fromme P). Wiley-Blackwell (2008).

126. Allen JP, Williams JC. Reaction centers from purple bacteria. In: Photosynthetic Protein Complexes (ed Fromme P). Wiley-Blackwell (2008).

127. Krogh A, Larsson B, von Heijne G, Sonnhammer EL. Predicting transmembrane protein topology with a hidden Markov model: application to complete genomes. J. Mol. Biol. 305, 567-580 (2001).

128. Søndergaard D, Pedersen CN, Greening C. HydDB: A web tool for hydrogenase classification and analysis. Sci. Rep. 6, 34212 (2016).

129. Chen IA, et al. IMG/M v.5.0: an integrated data management and comparative analysis system for microbial genomes and microbiomes. Nucl. Acids Res. 47, D666-d677 (2019). 
1301 130. Mukherjee S, et al. Genomes OnLine database (GOLD) v.7: updates and new features. Nucl. Acids Res. 47, D649-d659 (2019).

131. Kits KD, Klotz MG, Stein LY. Methane oxidation coupled to nitrate reduction under hypoxia by the Gammaproteobacterium Methylomonas denitrificans, sp. nov. type strain FJG1. Environ. Microbiol. 17, 3219-3232 (2015).

132. Tavormina PL, Orphan VJ, Kalyuzhnaya MG, Jetten MS, Klotz MG. A novel family of functional operons encoding methane/ammonia monooxygenase-related proteins in gammaproteobacterial methanotrophs. Environ. Microbiol. Rep. 3, 91-100 (2011).

133. Vorobev A, et al. Genomic and transcriptomic analyses of the facultative methanotroph Methylocystis sp. strain SB2 grown on methane or ethanol. Appl. Environ. Microbiol. 80, 3044-3052 (2014). 
Figure 1 bioRxiv preprint doi: https://doi.org/10.1101/2020.09.14.296780; this version posted September 14, 2020. The copyright holder for thlis preprint

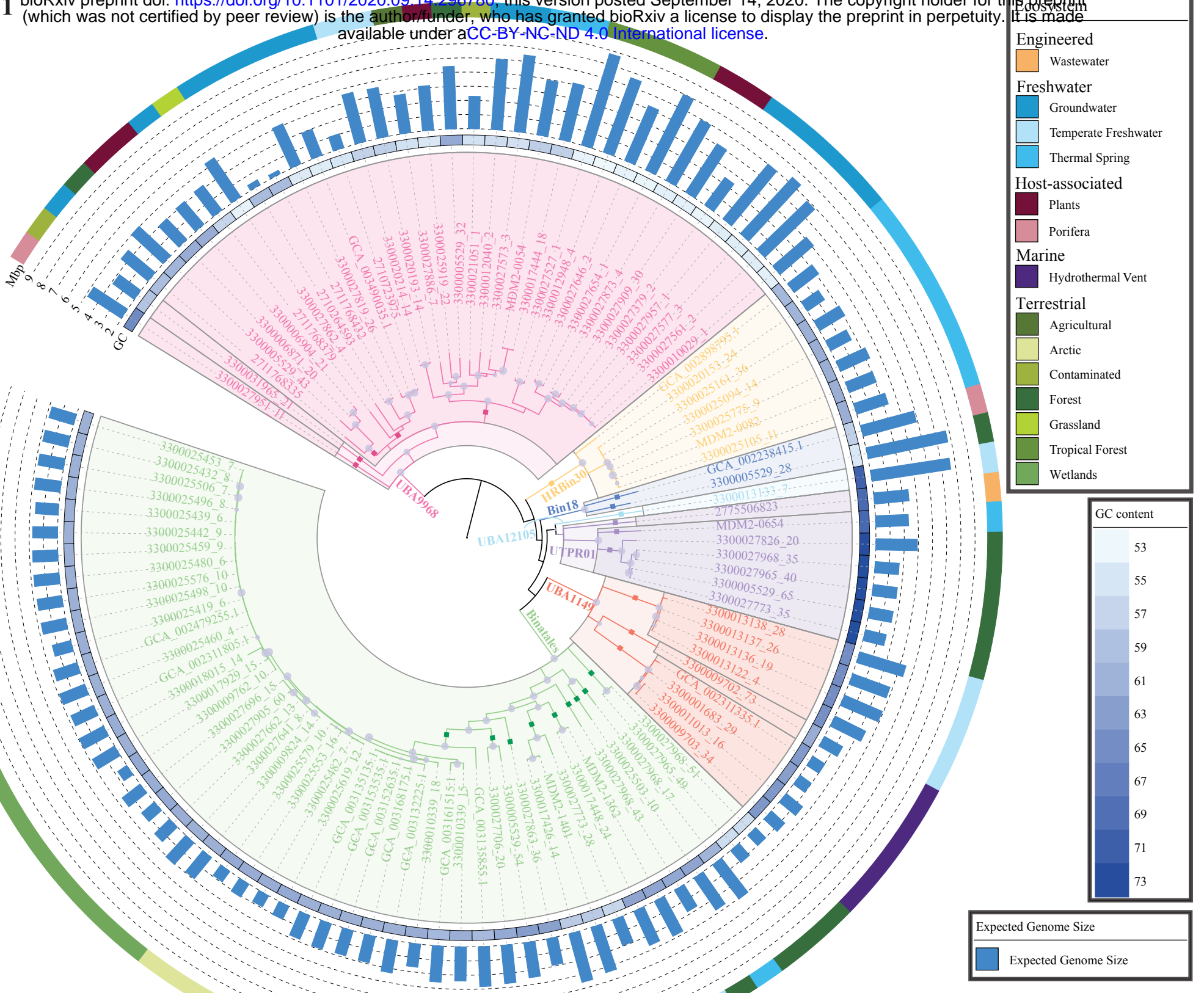

0.1 


\section{Figure 2}

A. C1 metabolism in the Binatota

\begin{tabular}{|c|c|c|c|c|c|c|c|c|c|c|c|c|c|c|c|c|c|c|c|c|c|c|c|c|c|c|}
\hline & & $\begin{array}{l}\text { Me } \\
\text { oxi }\end{array}$ & $\begin{array}{l}\text { han } \\
\text { atio }\end{array}$ & & \multirow{2}{*}{\multicolumn{5}{|c|}{$\begin{array}{l}\text { Methanol } \\
\text { oxidation }\end{array}$}} & \multirow{2}{*}{\multicolumn{12}{|c|}{ Methylamine oxidation }} & \multicolumn{4}{|c|}{$\begin{array}{c}\text { Methylated } \\
\text { sulfur } \\
\text { compounds }\end{array}$} & 嶽 \\
\hline & \multicolumn{4}{|c|}{ рMMO } & & & & & & & & & & & & & & & & & & \multicolumn{3}{|c|}{$\begin{array}{l}\text { Dimethyl } \\
\text { sulfone, } \\
\text { DMS }\end{array}$} & \multirow{2}{*}{\multicolumn{2}{|c|}{ 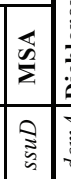 }} \\
\hline & 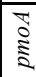 & $\begin{array}{l}\text { है } \\
\text { है }\end{array}$ & \begin{tabular}{|l|} 
\\
0 \\
$\vdots$ \\
$\vdots$
\end{tabular} & $\begin{array}{l}\text { 尺े } \\
\vdots \\
\vdots\end{array}$ & 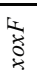 & ج্টة & ర్ర & §ิ & ミ & 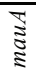 & 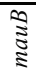 & 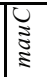 & 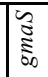 & 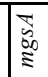 & $\begin{array}{l}0 \\
\vdots \\
: 0 \\
\equiv\end{array}$ & $\begin{array}{l}0 \\
0 \\
:\end{array}$ & $\begin{array}{l}\mathbb{Z} \\
0 \\
0 \\
3\end{array}$ & & $=$ & $\begin{array}{l}\mathbb{Z} \\
\mathbb{\infty} \\
\Xi\end{array}$ & $\begin{array}{l}\stackrel{0}{0} \\
\cong \\
\cong\end{array}$ & 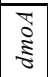 & \begin{tabular}{|l|} 
\\
5 \\
5
\end{tabular} & 8 & & \\
\hline \multicolumn{27}{|l|}{ Bin18 } \\
\hline \multicolumn{27}{|l|}{ Binatales } \\
\hline \multicolumn{27}{|l|}{ HRBin30 } \\
\hline \multicolumn{27}{|l|}{ UBA1149 } \\
\hline \multicolumn{27}{|c|}{ UBA12105 } \\
\hline \multicolumn{27}{|l|}{ UBA9968 } \\
\hline UTPR01 & & & & & & & & & & & & & & & & & & & & & & & & & & \\
\hline
\end{tabular}

Methylamine gmas $\downarrow$ 55-methyl-L-glutamine $m g s A B C \downarrow$

N-methyl-L-glutamate $n g d A B C D \downarrow$ Formaldehyde $\underbrace{\text { SsuD }}_{\text {Sulfite }}$

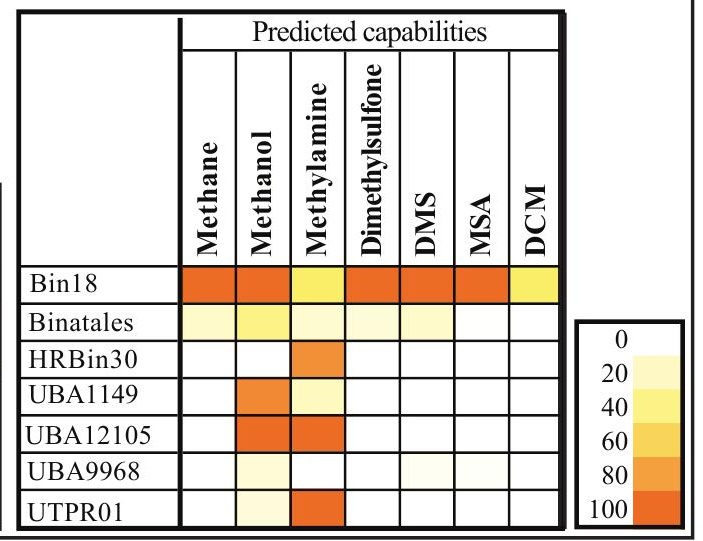

)

Actinobacteria/ SAR324

Lambdaproteobacteria) group

TUSC (uncultured cluster 2 group)

7

e

\section{B. Methanol dehydrogenase}

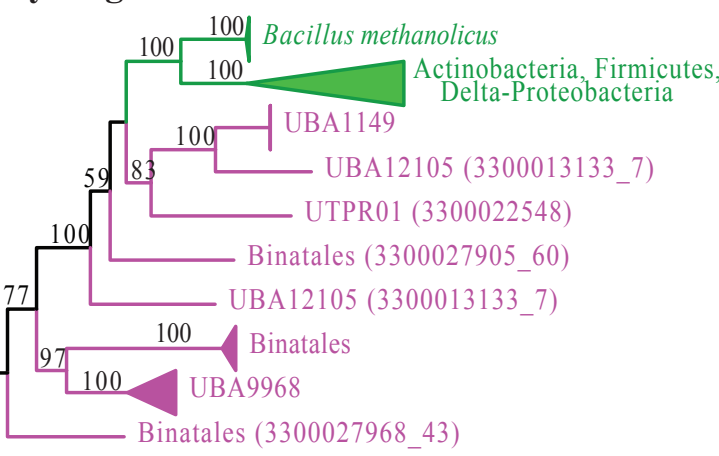

C. pMMO genomic organization

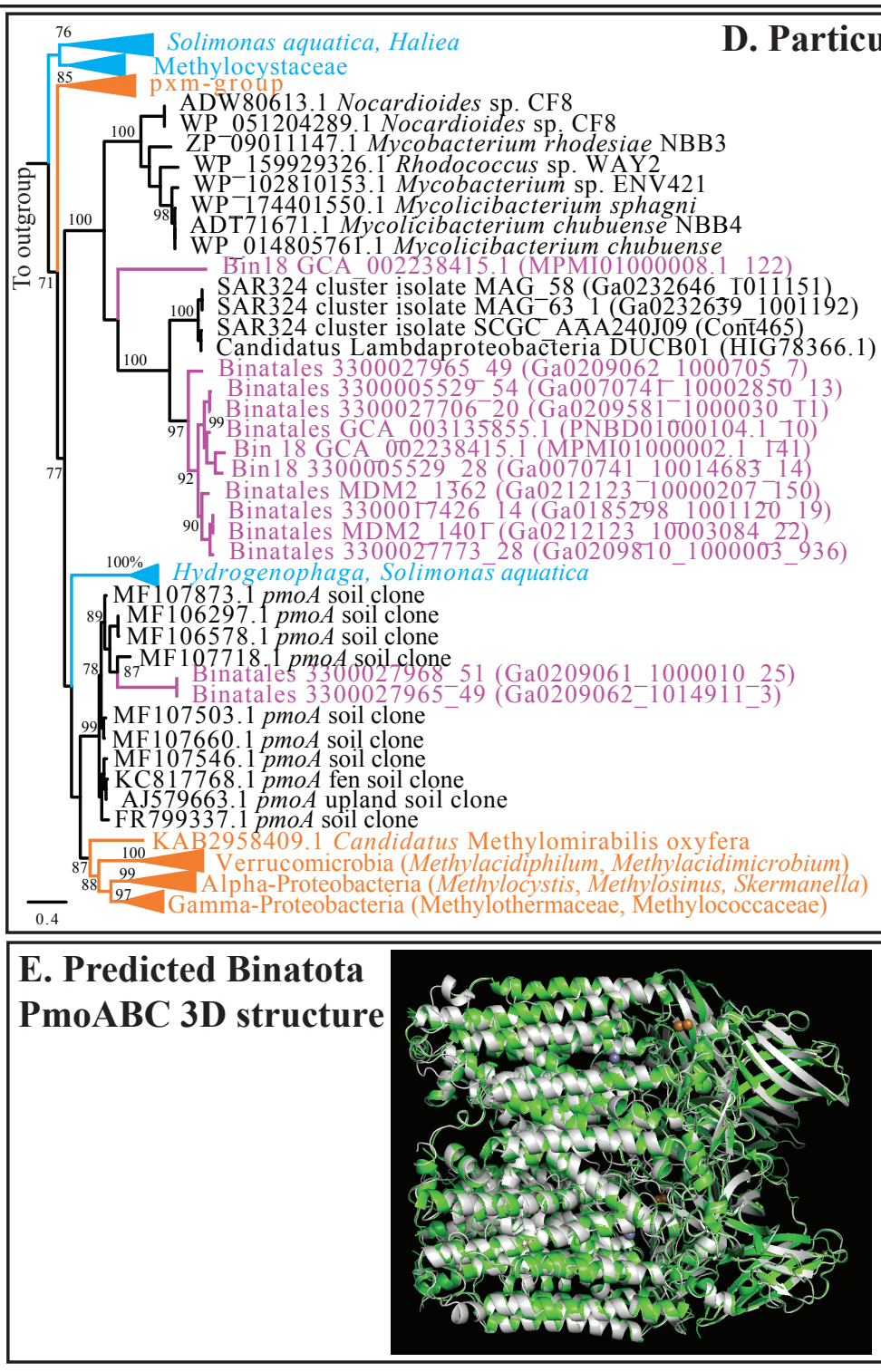
subunit A

$$
\text { (Lam }
$$$$
\mid
$$

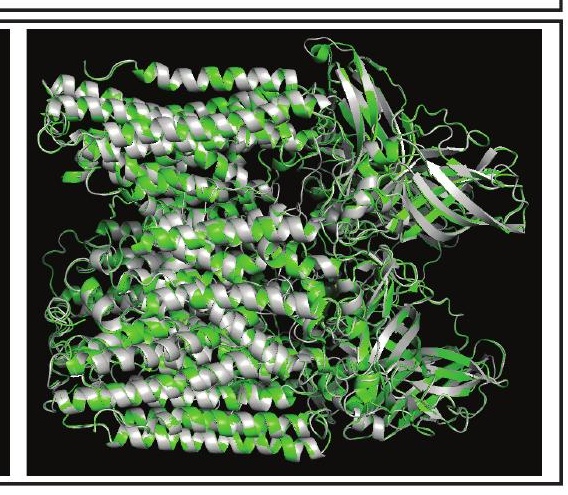

5 genomes

7 genomes 1 genome

$$
\begin{array}{|c|}
\hline p m o C \\
\hline \hline p m o C \\
\hline
\end{array}
$$

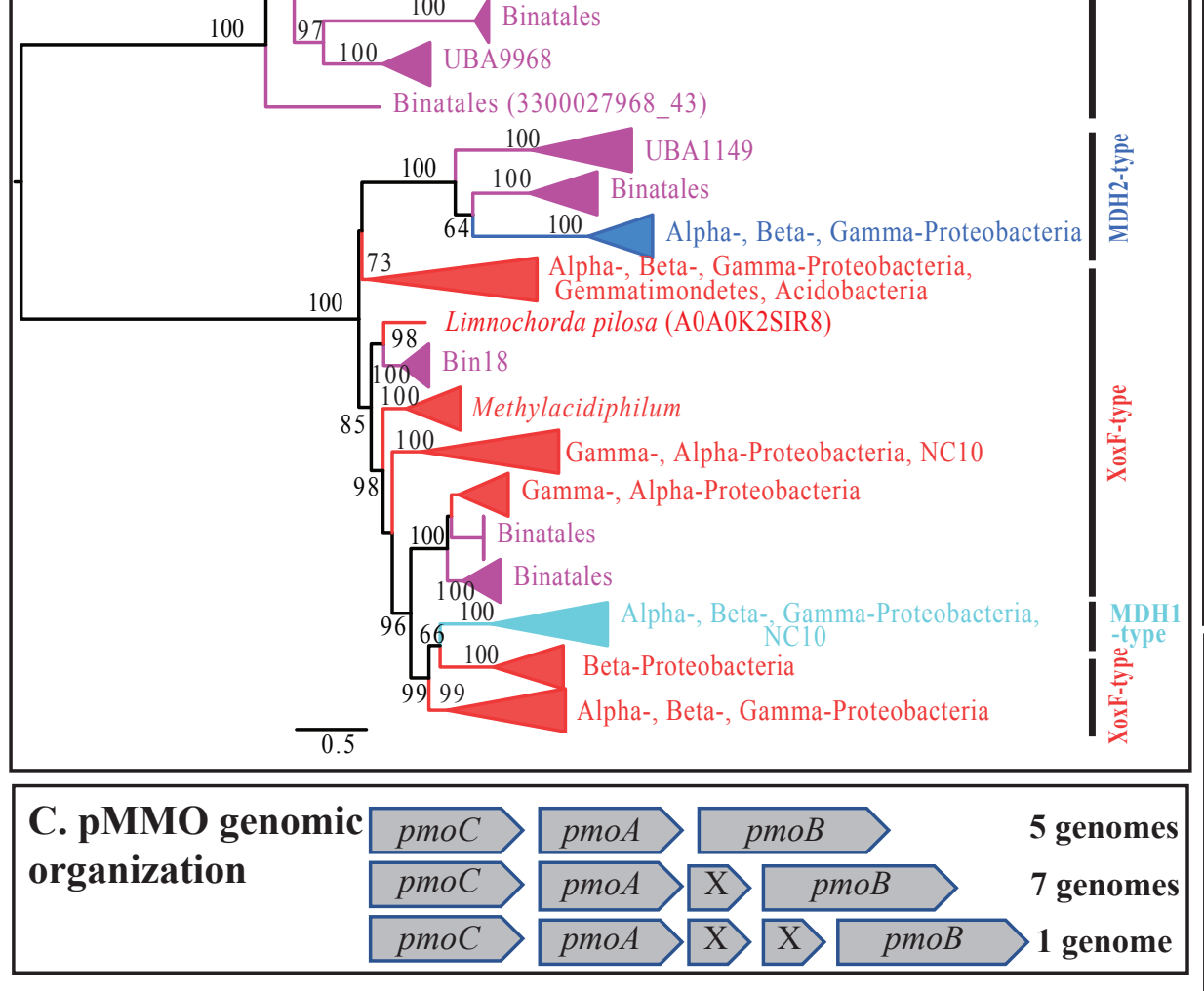




\begin{tabular}{|c|c|c|c|c|c|c|c|c|c|c|c|c|c|c|}
\hline \multirow{3}{*}{\multicolumn{2}{|c|}{$\mathbf{A}_{1}$}} & \multicolumn{11}{|c|}{ Formaldehyde oxidation } & \multirow{2}{*}{ 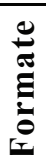 } & \\
\hline & & \multicolumn{4}{|c|}{ Thiol-dependent } & \multicolumn{3}{|c|}{ H4F-linked } & & \multicolumn{3}{|c|}{$\begin{array}{c}\text { GSH- } \\
\text { dependent }\end{array}$} & & \\
\hline & & 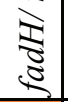 & $\underset{\frac{\Xi}{\Xi}}{\Xi}$ & $\begin{array}{l}\infty \\
\frac{1}{2} \\
\vdots\end{array}$ & $\begin{array}{l}\text { 侪 } \\
\vdots\end{array}$ & $\underset{0}{2}$ & $\begin{array}{l}? \\
\vdots \\
\vdots\end{array}$ & $\stackrel{5}{S}$ & $\underset{d}{\frac{\pi}{2}}$ & $\frac{\pi}{\infty}$ & ह & 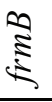 & $\stackrel{5}{\vdots}$ & \\
\hline & Bin 18 & & & & & & & & & & & & & \\
\hline & Binatales & & & & & & & & & & & & & \\
\hline & HRBin30 & & & & & & & & & & & & & 0 \\
\hline & UBA1149 & & & & & & & & & & & & & 20 \\
\hline & UBA12105 & & & & & & & & & & & & & 40 \\
\hline & UBA9968 & & & & & & & & & & & & & $\begin{array}{l}60 \\
80\end{array}$ \\
\hline & UTPR01 & & & & & & & & & & & & & 100 \\
\hline
\end{tabular}

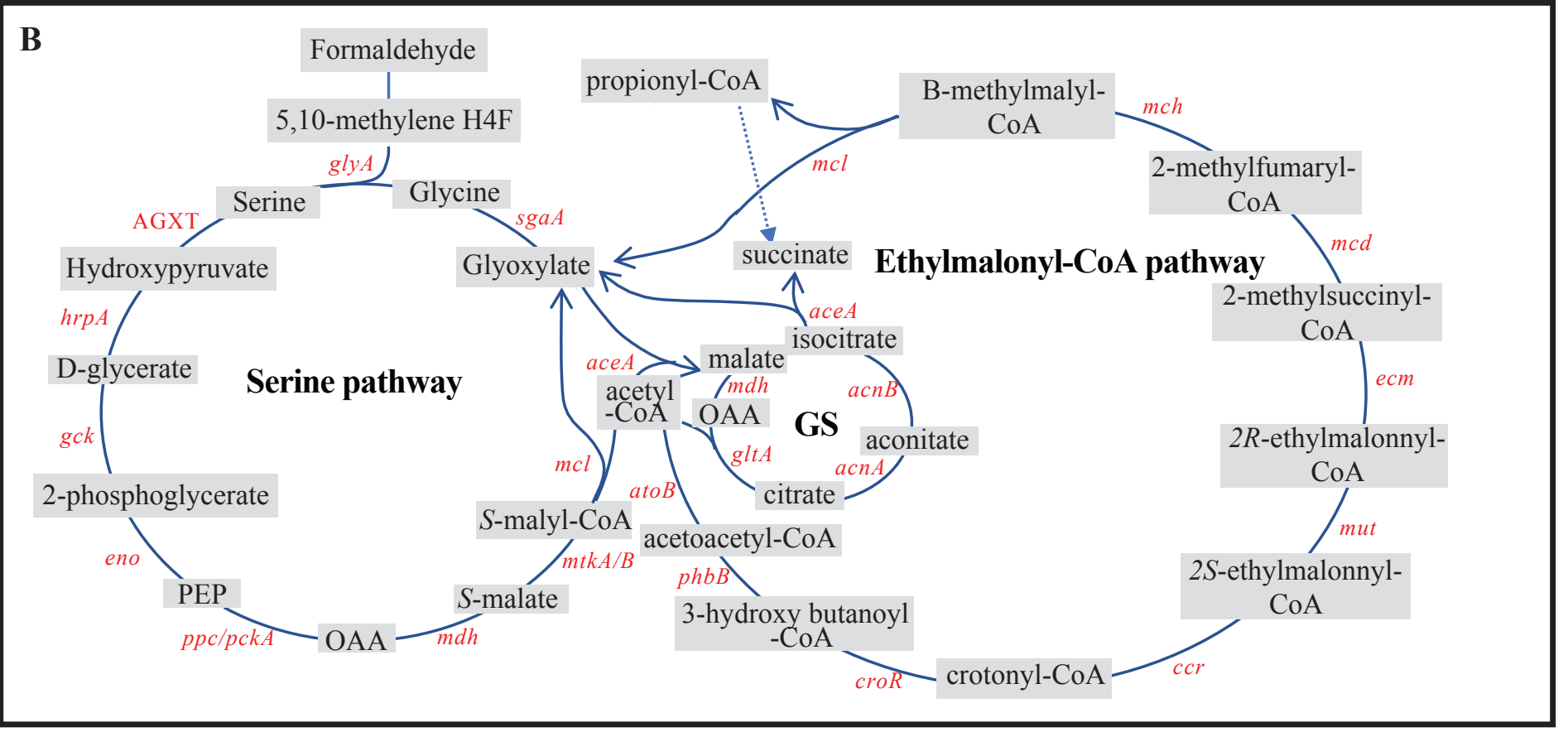

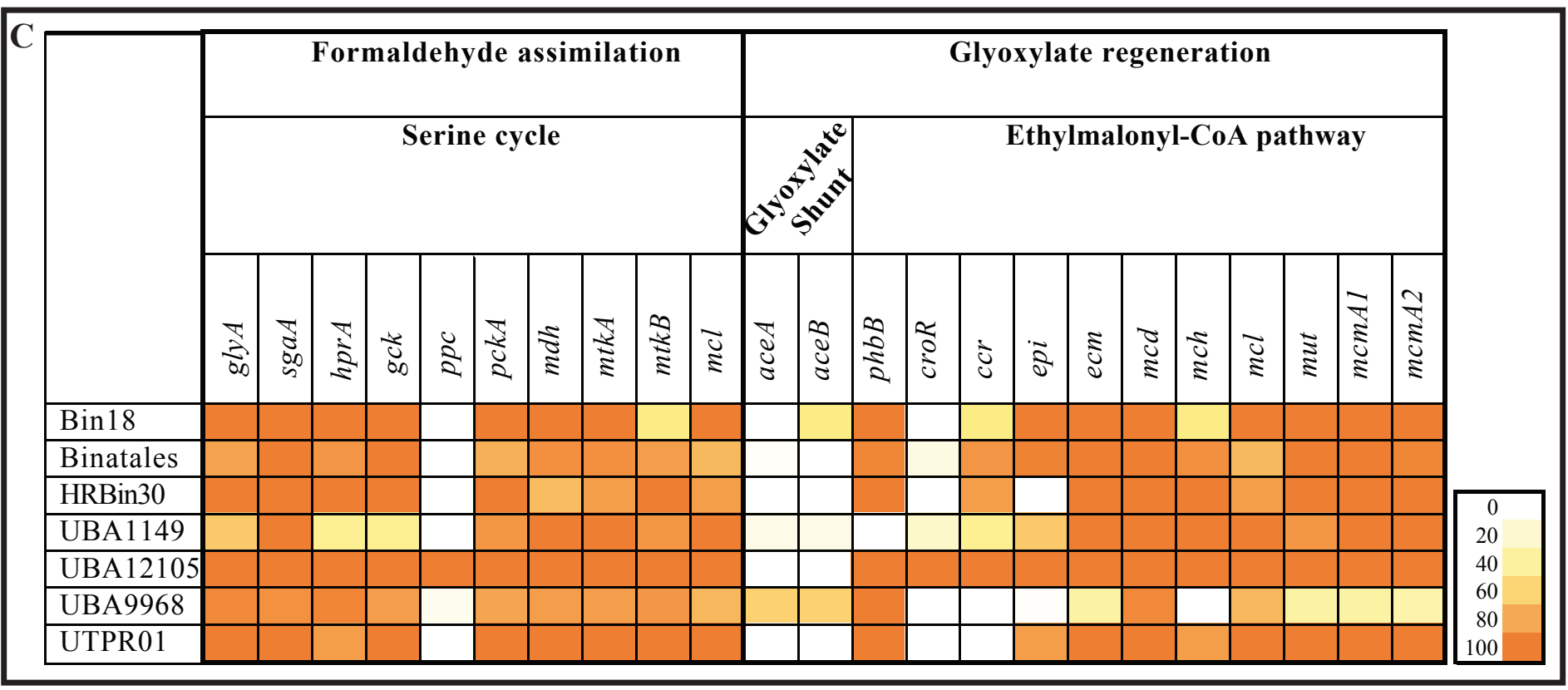


bioRxiv preprint doi: https://doi.org/10.1101/2020.09.14.296780; this version posted September 14, 2020. The copyright holder for this preprint (which was not certified by peer review) is the author/funder, who has granted bioRxiv a license to display the preprint in perpetuity. It is made Figure $4 \begin{aligned} & \text { (which was not certified by peer review) is the author/funder, who has granted bioRxiv a license to disp } \\ & \text { available under aCC-BY-NC-ND } 4.0 \text { International license. }\end{aligned}$

\begin{tabular}{|c|c|c|c|c|c|c|c|c|c|c|c|c|c|c|c|c|c|c|c|c|c|c|c|}
\hline \multirow{5}{*}{\multicolumn{2}{|c|}{$\mathbf{A}$}} & \multicolumn{10}{|c|}{ (Halo)Alkane degradation to alcohols } & \multicolumn{6}{|c|}{ Alcohol oxidation to FA } & & & & & & \\
\hline & & \multicolumn{6}{|c|}{ Short chain alkanes } & \multirow{3}{*}{\multicolumn{2}{|c|}{ 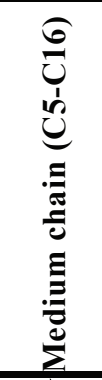 }} & \multirow{3}{*}{ 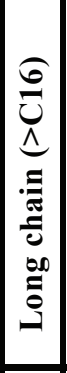 } & \multirow{3}{*}{ 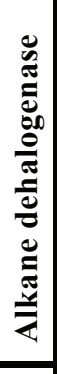 } & \multirow{3}{*}{\multicolumn{2}{|c|}{ 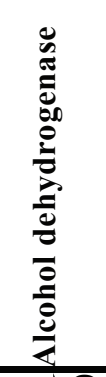 }} & \multirow{3}{*}{\multicolumn{2}{|c|}{ "ृ }} & \multirow{3}{*}{ 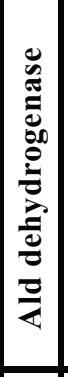 } & \multirow{3}{*}{ 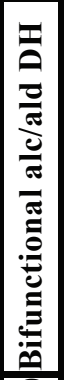 } & & & & & & \\
\hline & & \multirow{2}{*}{\multicolumn{3}{|c|}{ 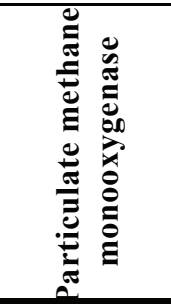 }} & \multirow{2}{*}{\multicolumn{3}{|c|}{ 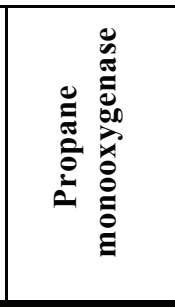 }} & & & & & & & & & & & & \multicolumn{4}{|c|}{\begin{tabular}{|c} 
Alkane \\
dehgradation \\
capabilities
\end{tabular}} & \\
\hline & & & & & & & & & & & & & & & & & & & \multirow{2}{*}{ 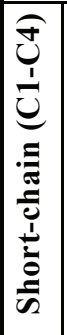 } & \multirow{2}{*}{ 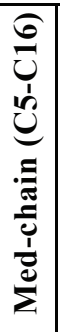 } & \multirow{2}{*}{ 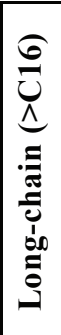 } & \multirow{2}{*}{ 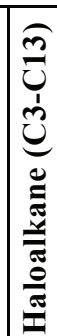 } & \\
\hline & & $\begin{array}{l}\mathbb{Z} \\
0 \\
\vdots \\
0\end{array}$ & 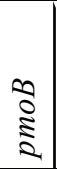 & 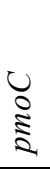 & $\begin{array}{l}\mathbb{Z} \\
\mathbb{E}\end{array}$ & $\stackrel{\text { है }}{\text { है }}$ & ミ & $\frac{\infty}{3}$ & 艿 & $\mathbb{\Xi}$ & $\begin{array}{l}\mathbb{Z} \\
\mathbb{Z}\end{array}$ & శ్ & 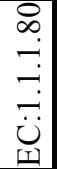 & క్ & $\begin{array}{l}\text { है } \\
\text { है }\end{array}$ & 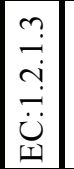 & 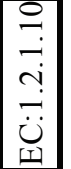 & & & & & & \\
\hline & Bin18 & & & & & & & & & & & & & & & & & Bin18 & & & & & \\
\hline & Binatales & & & & & & & & & & & & & & & & & Binatales & & & & & \\
\hline & HRBin30 & & & & & & & & & & & & & & & & & HRBin30 & & & & & 0 \\
\hline & UBA1149 & & & & & & & & & & & & & & & & & \begin{tabular}{|l} 
UBA1149 \\
\end{tabular} & & & & & 20 \\
\hline & UBA12105 & & & & & & & & & & & & & & & & & UBA12105 & & & & & $\begin{array}{l}40 \\
60\end{array}$ \\
\hline & UBA9968 & & & & & & & & & & & & & & & & & \begin{tabular}{|l|} 
UBA9968 \\
\end{tabular} & & & & & $\begin{array}{l}60 \\
80\end{array}$ \\
\hline & UTPR01 & & & & & & & & & & & & & & & & & \begin{tabular}{|l|} 
UTPR01 \\
\end{tabular} & & & & & 100 \\
\hline
\end{tabular}

\begin{tabular}{|c|c|c|c|c|c|c|c|c|c|c|c|c|c|c|c|c|c|c|c|c|c|c|c|c|c|c|c|c|c|c|c|c|c|c|c|c|}
\hline \multirow{3}{*}{\multicolumn{2}{|c|}{ B }} & \multicolumn{6}{|c|}{$\begin{array}{c}\text { Activation (CoA- } \\
\text { ligation) }\end{array}$} & \multicolumn{11}{|c|}{ Propionyl-CoA degradation } & \multicolumn{7}{|c|}{$\begin{array}{l}\text { Butanoyl-CoA/med chain } \\
\text { fatty acyl-CoA degradation }\end{array}$} & \multicolumn{5}{|c|}{ Long chain FA } & \multicolumn{6}{|c|}{ Haloacid degradation } \\
\hline & & \multicolumn{2}{|c|}{ 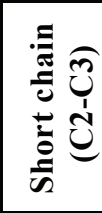 } & \multicolumn{3}{|c|}{ 竝 } & 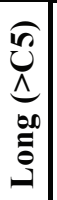 & \multicolumn{3}{|c|}{$\begin{array}{l}\text { Methyl- } \\
\text { malonyl- } \\
\text { CoA cycle }\end{array}$} & \multicolumn{5}{|c|}{$\begin{array}{l}\text { Glyoxylate } \\
\text { assimilation }\end{array}$} & \multicolumn{3}{|c|}{$\begin{array}{c}\text { 2-methyl } \\
\text { citrate } \\
\text { cycle }\end{array}$} & \multicolumn{2}{|c|}{ 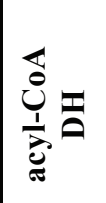 } & \multicolumn{2}{|c|}{ 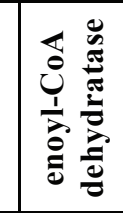 } & \multicolumn{2}{|c|}{ 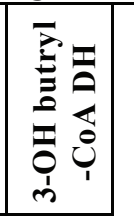 } & \multirow{2}{*}{ 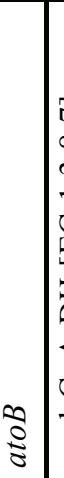 } & \multicolumn{3}{|c|}{ 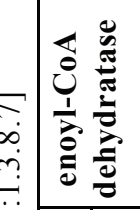 } & \multirow{2}{*}{ 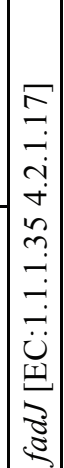 } & \multirow{2}{*}{ 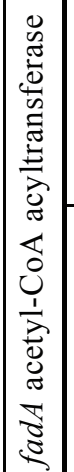 } & \multicolumn{2}{|c|}{ 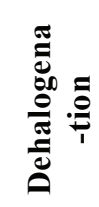 } & \multicolumn{4}{|c|}{$\begin{array}{c}\text { Hydroxyacid } \\
\text { oxidase }\end{array}$} \\
\hline & & 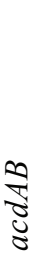 & $\hat{y}$ & $\begin{array}{l}\pi \\
8\end{array}$ & $\frac{8}{8}$ & 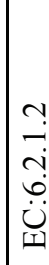 & 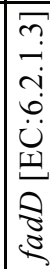 & 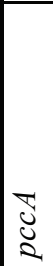 & $\tilde{3}$ & $\cong$ & $\cong$ & 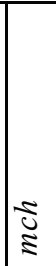 & $\stackrel{\Xi}{\Xi}$ & §ँ & ปี & מُ & : & ڤ્ & 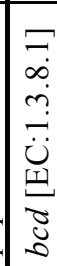 & 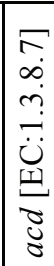 & $\mid$ & 3 & 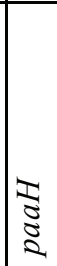 & $\begin{array}{l}\infty \\
\vdots \\
2 \\
2\end{array}$ & & 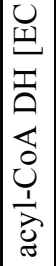 & $\mid \begin{array}{l}1 \\
0 \\
0 \\
2\end{array}$ & & & & $\frac{\pi}{2}$ & 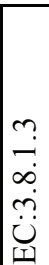 & $\frac{\theta}{\partial}$ & $\frac{1}{0}$ & $\frac{1}{\Delta}$ & 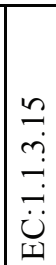 \\
\hline & Bin18 & & & & & & & & & & & & & & & & & & & & & & & & & & & & & & & & & & & \\
\hline & Binatales & & & & & & & & & & & & & & & & & & & & & & & & & & & & & & & & & & & \\
\hline & HRBin30 & & & & & & & & & & & & & & & & & & & & & & & & & & & & & & & & & & & \\
\hline & UBA1149 & & & & & & & & & & & & & & & & & & & & & & & & & & & & & & & & & & & \\
\hline & UBA12105 & & & & & & & & & & & & & & & & & & & & & & & & & & & & & & & & & & & \\
\hline & \begin{tabular}{|l|} 
UBA99668 \\
\end{tabular} & & & & & & & & & & & & & & & & & & & & & & & & & & & & & & & & & & & \\
\hline & UTPR01 & & & & & & & & & & & & & & & & & & & & & & & & & & & & & & & & & & & \\
\hline
\end{tabular}

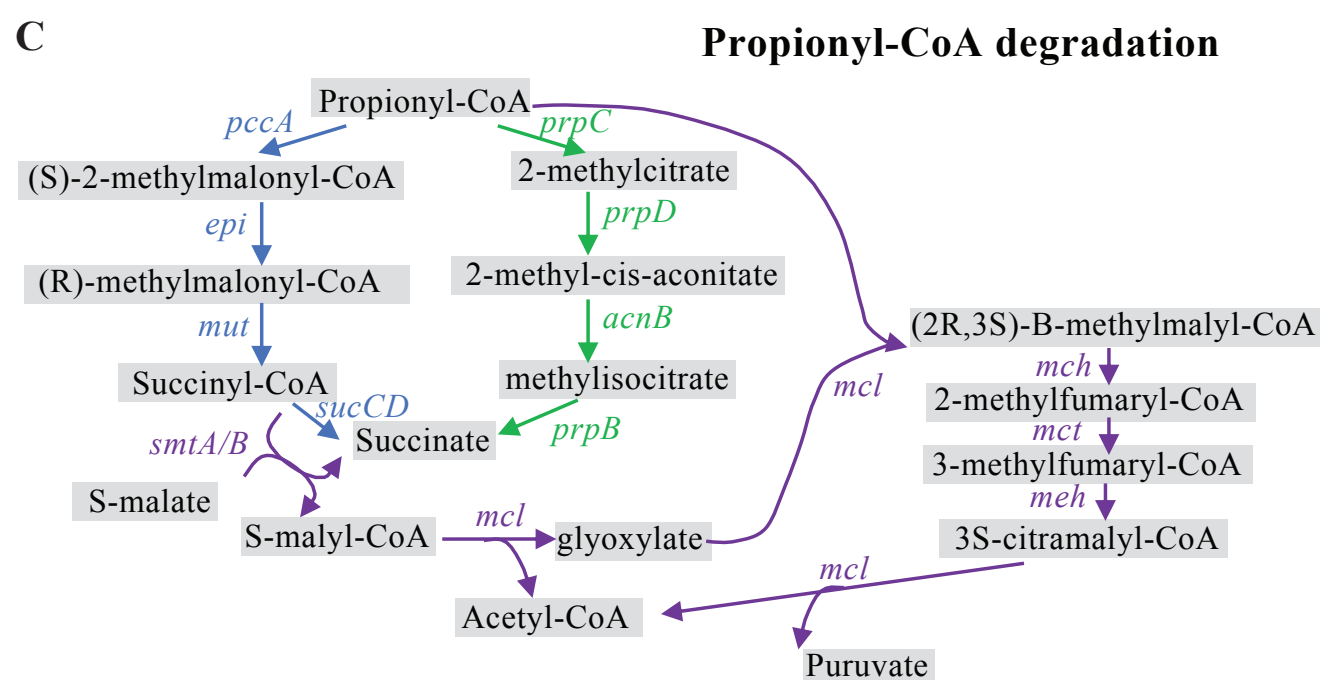


bioRxiv preprint doi: https://doi.org/10.1101/2020.09.14.296780; this version posted September 14,2020 . The copyright holder for this preprint Figure 5 (which was not certified by peer review) is the author/funder, who has granted bioRxiv a license to display the preprint in perpetuity. It is made available under aCC-BY-NC-ND 4.0 International license.

\begin{tabular}{|c|c|c|c|c|c|c|c|c|c|c|c|c|c|c|c|c|c|c|c|c|c|c|c|c|c|c|c|c|c|}
\hline \multirow{4}{*}{ A } & \multirow{4}{*}{ 芠 } & \multirow{4}{*}{$\begin{array}{l}= \\
\bar{x} \\
\frac{\partial}{a} \\
\bar{\Xi} \\
\dot{u}\end{array}$} & \multirow{2}{*}{\multicolumn{9}{|c|}{ Quinone-cytochrome $\mathrm{C}$ reductase }} & \multirow{2}{*}{\multicolumn{9}{|c|}{$\begin{array}{c}\text { Cytochrome C oxidase (complex } \\
\text { IV) }\end{array}$}} & \multirow{3}{*}{\multicolumn{5}{|c|}{$\begin{array}{c}\begin{array}{c}\text { Electron transfer } \\
\text { proteins }\end{array} \\
\text { Cyt } \mathrm{C} \text { classes }\end{array}$}} & \multirow{3}{*}{\multicolumn{3}{|c|}{$\begin{array}{l}\text { [NiFe] } \\
\text { H2ase }\end{array}$}} & \\
\hline & & & & & & & & & & & & & & & & & & & & & & & & & & & & & \\
\hline & & & \multicolumn{6}{|c|}{ ACIII } & \multicolumn{3}{|c|}{ Complex III } & \multicolumn{4}{|c|}{ family $A$} & \multicolumn{3}{|c|}{ family $\mathbf{C}$} & \multicolumn{2}{|c|}{ Cyt $b d$} & & & & & & & & & \\
\hline & & & $\underset{J}{\mathbb{Z}}$ & $\underset{0}{0}$ & 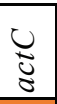 & $\underset{\Xi}{0}$ & 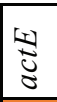 & $\begin{array}{l}0 \\
0 \\
0 \\
\end{array}$ & $\tilde{\approx}$ & 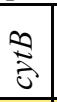 & 范 & $\begin{array}{l}\pi \\
0 \\
0\end{array}$ & 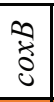 & 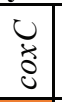 & $\begin{array}{l}0 \\
0 \\
0 \\
0 \\
0\end{array}$ & \begin{tabular}{|l|} 
\\
\\
0 \\
0
\end{tabular} & $\begin{array}{l}0 \\
\vdots \\
0 \\
0\end{array}$ & $\begin{array}{l}0 \\
0 \\
0 \\
0\end{array}$ & $\begin{array}{l}\pi \\
0 \\
0\end{array}$ & 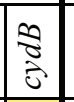 & $\sum_{\Xi}^{\ominus}$ & $\varrho$ & $\theta$ & $\sqsupseteq$ & $\Xi$ & 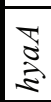 & 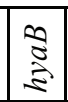 & 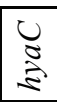 & \\
\hline Bin18 & & & & & & & & & & & & & & & & & & & & & & & & & & & & & \\
\hline Binatales & & & & & & & & & & & & & & & & & & & & & & & & & & & & & \\
\hline HRBin 30 & & & & & & & & & & & & & & & & & & & & & & & & & & & & & 0 \\
\hline UBA1149 & & & & & & & & & & & & & & & & & & & & & & & & & & & & & 20 \\
\hline UBA12105 & & & & & & & & & & & & & & & & & & & & & & & & & & & & & $\begin{array}{l}40 \\
60\end{array}$ \\
\hline UBA9968 & & & & & & & & & & & & & & & & & & & & & & & & & & & & & 80 \\
\hline UTPR01 & & & & & & & & & & & & & & & & & & & & & & & & & & & & & 100 \\
\hline
\end{tabular}
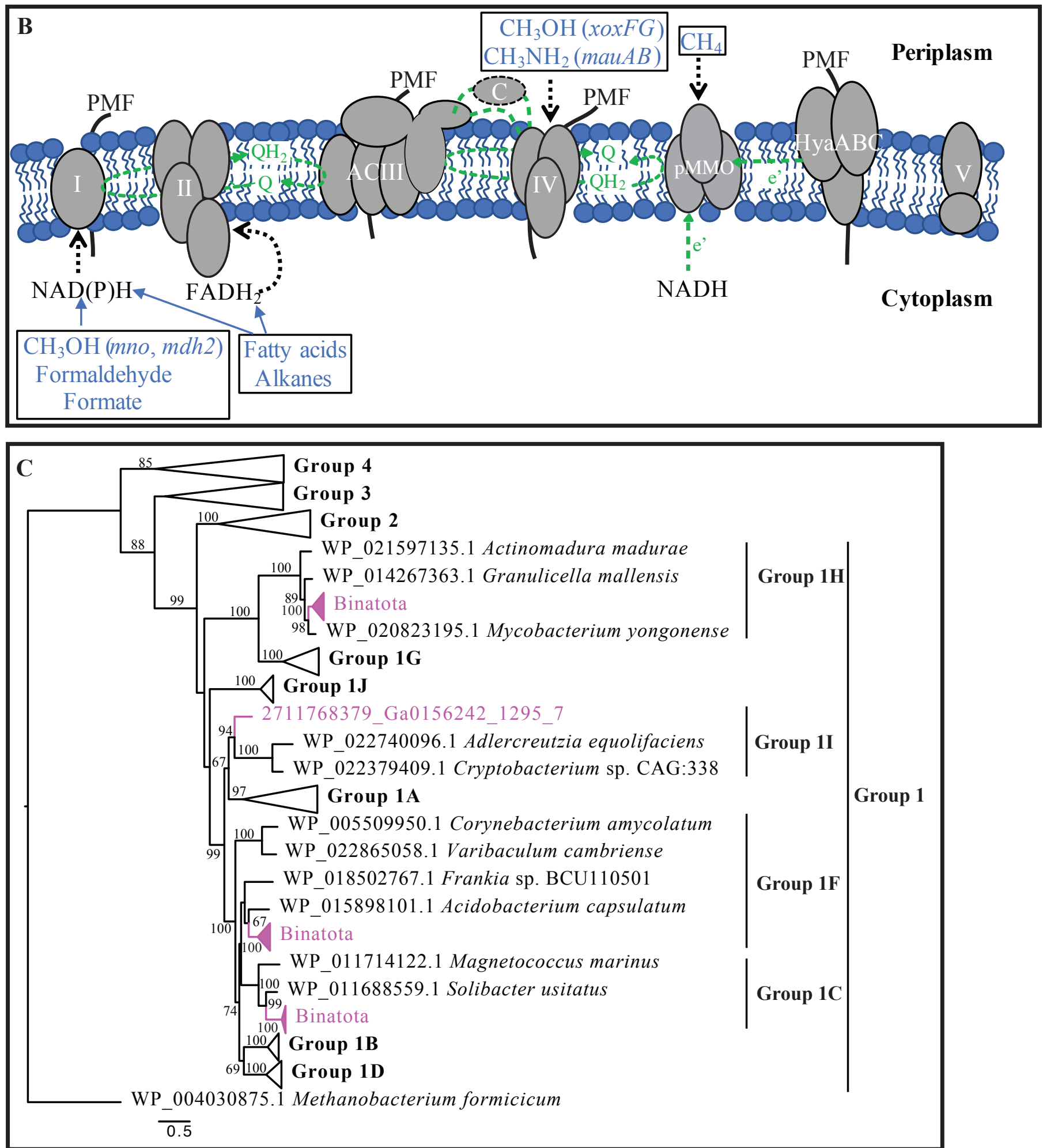


\begin{tabular}{|c|c|c|c|c|c|c|c|c|c|c|c|c|c|c|c|c|c|c|}
\hline & \multicolumn{7}{|c|}{ Chlorophyllide $a$ biosynthesis } & \multicolumn{4}{|c|}{ Bchl $a$ biosynthesis } & \multicolumn{6}{|c|}{$\operatorname{Bchl} c$, and $d$ biosynthesis } & \\
\hline & 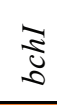 & $\underset{\Xi}{J}$ & $\frac{1}{\mathfrak{J}}$ & $\underset{0}{\stackrel{J}{J ~}}$ & $\underset{J}{\vdots}$ & 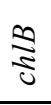 & 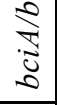 & $\frac{1}{8}$ & 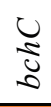 & $\underset{0}{\stackrel{0}{U}}$ & 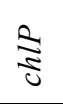 & $\underset{\Xi}{U}$ & $\frac{\mathfrak{s}}{\mathfrak{s}}$ & 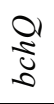 & $\underset{\Xi}{0}$ & 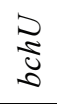 & $\frac{1}{3}$ & \\
\hline \multicolumn{18}{|l|}{ Bin 18} & \\
\hline \multicolumn{19}{|l|}{ Binatales } \\
\hline HRBin30 & & & & & & & & & & & & & & & & & & \multirow{5}{*}{$\begin{array}{r}0 \\
20 \\
40 \\
60 \\
80 \\
100\end{array}$} \\
\hline UBA1149 & & & & & & & & & & & & & & & & & & \\
\hline UBA1210 & & & & & & & & & & & & & & & & & & \\
\hline UBA9968 & & & & & & & & & & & & & & & & & & \\
\hline UTPR01 & & & & & & & & & & & & & & & & & & \\
\hline
\end{tabular}

B

Protoporphyrin-IX bchI

Mg-Protoporphyrin-IX bchM

Mg-Protoporphyrin-IX

13-monomethyl ester

$$
\begin{aligned}
& b c h E \\
& b c h E \\
& b c h E
\end{aligned}
$$

3,8-divinyl protochlorophyllide a bchLNB

chlorophyllide $a_{2}$ $b c i A / b c i B / b c h X Y Z$

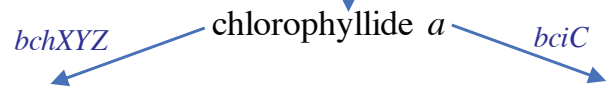

3-vinyl bacteriochlorophyllide $a$<smiles>[C]1[CH]CC1</smiles>

3-hydroxyethyl bacteriochlorophyllide $a$

$$
\text { bchC }
$$

bacteriochlorophyllide $a$

$$
b c h G \downarrow
$$

Geranylgeranyl bacteriochlorophyllide $a$

$$
b c h P
$$

Bacteriochlorophyll $a$ 8-ethyl-12-methyl-3-vinyl

bacteriochlorophyllide $d$ bchR

8,12-diethyl-3-vinyl

bacteriochlorophyllide $d$

$$
b \operatorname{ch} Q \downarrow
$$

12-ethyl-8-propyl-3-vinyl bacteriochlorophyllide $d$

$$
b c h Q \downarrow
$$

12-ethyl-8-isobutyl-3-vinyl bacteriochlorophyllide $d$
bchI magnesium chelatase subunit H [EC:6.6.1.1]

bchLNB light-independent protochlorophyllide reductase [EC:1.3.7.7]

bciA 3,8-divinyl protochlorophyllide a 8-vinyl-reductase [EC:1.3.7.75]

bciB 3,8-divinyl protochlorophyllide a 8-vinyl-reductase [EC:1.3.7.13]

bch $X Y Z$ 3,8-divinyl chlorophyllide a/chlorophyllide a reductase

bciC chlorophyllide a hydrolase [EC:3.1.1.100]

bch $\boldsymbol{F} \quad$ 3-vinyl bacteriochlorophyllide hydratase [EC:4.2.1.165]

bchC bacteriochlorophyllide a dehydrogenase [EC:1.1.1.396]

chlorophyll/bacteriochlorophyll a synthase [EC:2.5.1.62 2.5.1.133] geranylgeranyl diphosphate/geranylgeranyl-bacteriochlorophyllide a reductase [EC:1.3.1.83 1.3.1.111]

bch $\boldsymbol{R}$ bacteriochlorophyllide d C-12(1)-methyltransferase [EC:2.1.1.331]

bch $Q$ bacteriochlorophyllide d C-8(2)-methyltransferase [EC:2.1.1.332]

bch $\boldsymbol{V}$ 3-vinyl bacteriochlorophyllide hydratase [EC:4.2.1.169]

bch $\boldsymbol{U}$ bacteriochlorophyllide d C-20 methyltransferase [EC:2.1.1.333]

bacteriochlorophyll c synthase [EC:2.5.1.-]

Bacteriochlorophyll $c$

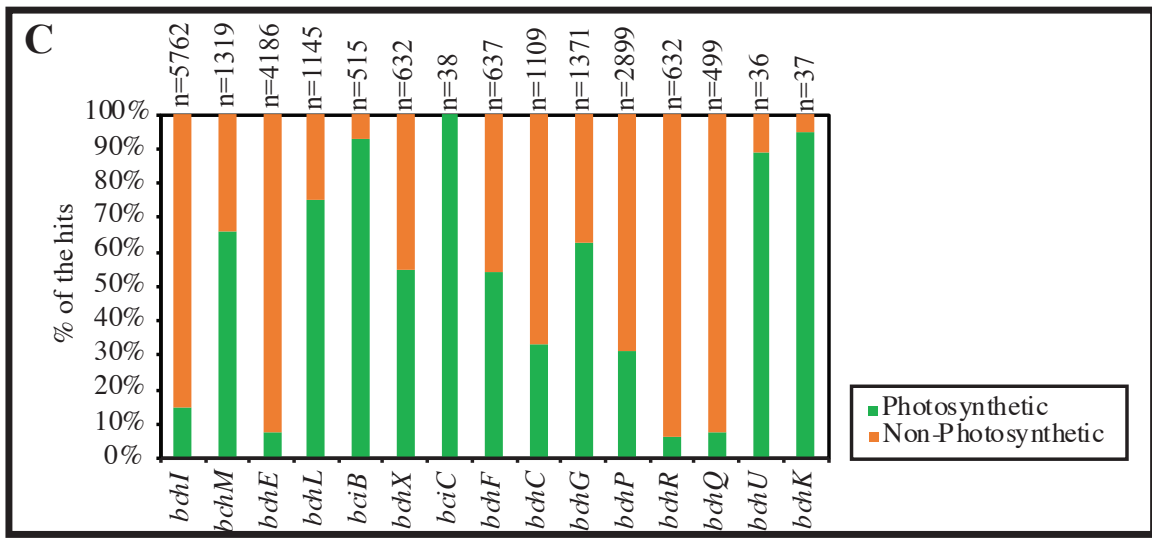


bioRxiv preprint doi: https://doi.org/10.1101/2020.09.14.296780; this version posted September 14,2020 . The copyright holder for this preprint

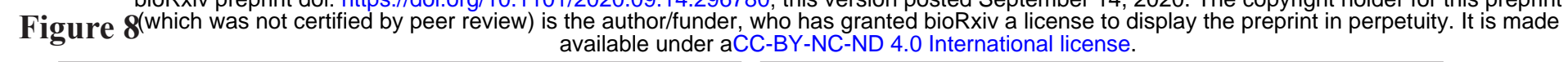
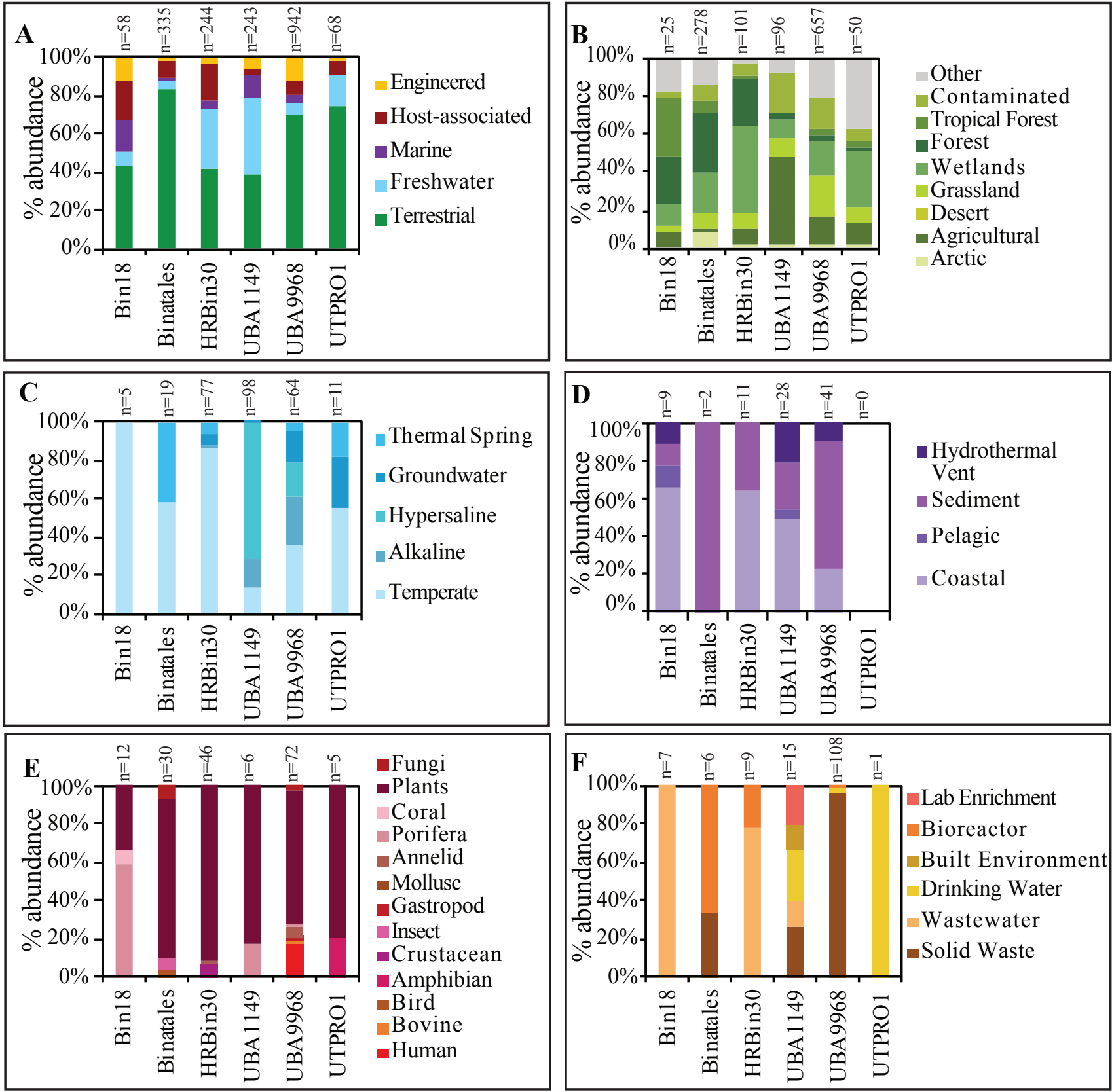
Figure 9

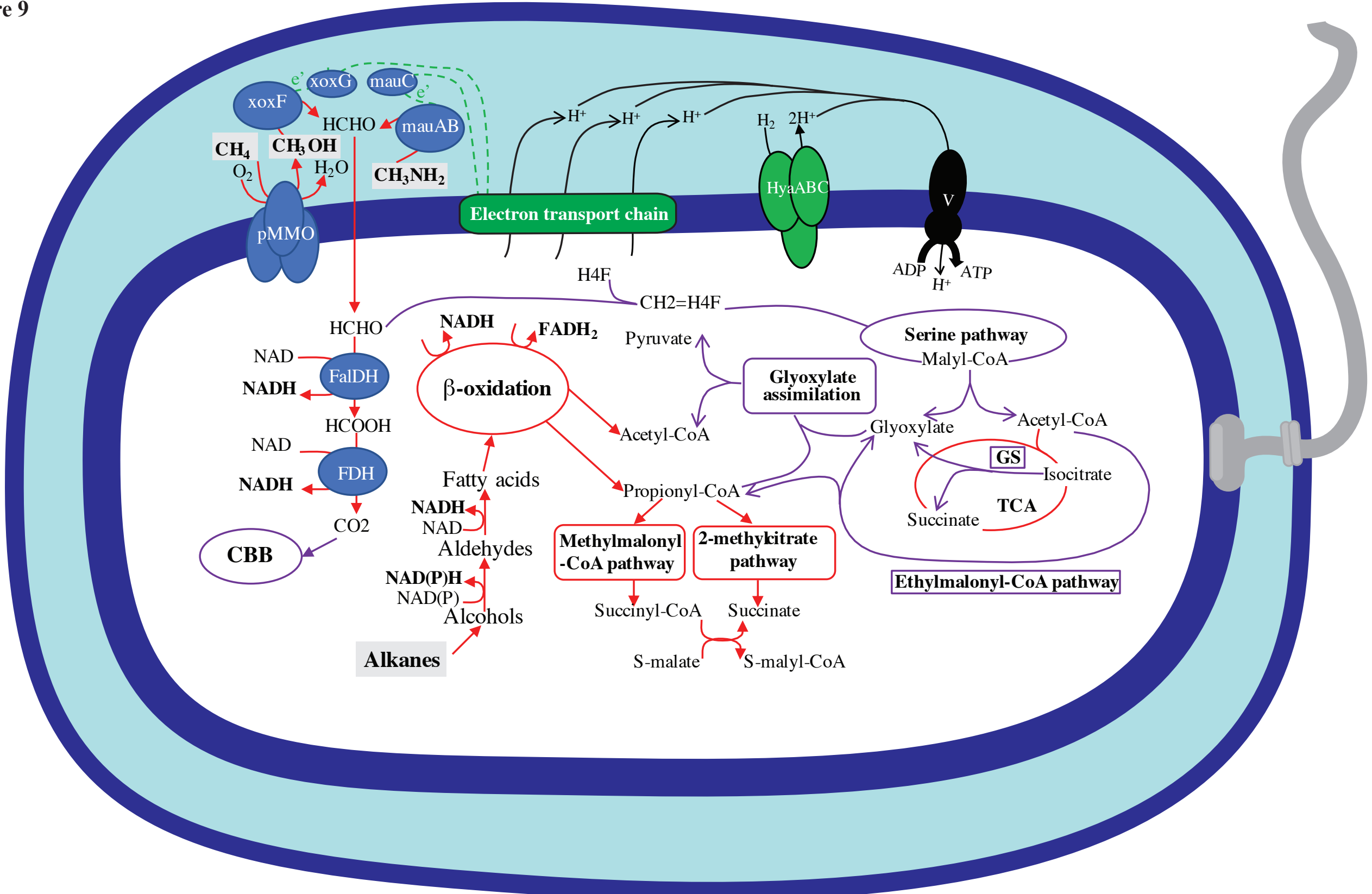

